Research Paper

\title{
Applicability of subcortical EEG metrics of synaptopathy to older listeners with impaired audiograms
}

\author{
Markus Garrett ${ }^{\mathrm{a},}{ }^{*}$, Sarah Verhulst ${ }^{\mathrm{b}, \mathrm{a}}$ \\ ${ }^{a}$ Medizinische Physik and Cluster of Excellence "Hearing4all", Department of Medical Physics and Acoustics, University of Oldenburg, Oldenburg, Germany \\ ${ }^{\mathrm{b}}$ Hearing Technology @ WAVES, Department of Information Technology, Ghent University, Ghent, Belgium
}

\section{A R T I C L E I N F O}

\section{Article history:}

Received 22 November 2018

Received in revised form

24 June 2019

Accepted 1 July 2019

Available online 2 July 2019

\section{Keywords:}

Auditory brainstem response

Envelope following response

Cochlear synaptopathy

Diagnostics

Sensorineural hearing loss

Deafferentation

\begin{abstract}
A B S T R A C T
Emerging evidence suggests that cochlear synaptopathy is a common feature of sensorineural hearing loss, but it is not known to what extent electrophysiological metrics targeting synaptopathy in animals can be applied to people, such as those with impaired audiograms. This study investigates the applicability of subcortical electrophysiological measures associated with synaptopathy, i.e., auditory brainstem responses (ABRs) and envelope following responses (EFRs), to older participants with highfrequency sloping audiograms. The outcomes of this study are important for the development of reliable and sensitive synaptopathy diagnostics in people with normal or impaired outer-hair-cell function. Click-ABRs at different sound pressure levels and EFRs to amplitude-modulated stimuli were recorded, as well as relative EFR and ABR metrics which reduce the influence of individual factors such as head size and noise floor level on the measures. Most tested metrics showed significant differences between the groups and did not always follow the trends expected from synaptopathy. Age was not a reliable predictor for the electrophysiological metrics in the older hearing-impaired group or young normal-hearing control group. This study contributes to a better understanding of how electrophysiological synaptopathy metrics differ in ears with healthy and impaired audiograms, which is an important first step towards unravelling the perceptual consequences of synaptopathy.
\end{abstract}

() 2019 Elsevier B.V. All rights reserved.

\section{Introduction}

The cochlea is a complex structure with many interdependent components that shape how we perceive sound. With age and/or exposure to noise or ototoxic agents, cochlear structures can deteriorate, yielding different degrees and manifestations of sensorineural hearing loss. Outer-hair-cell (OHC) damage is an important contributor to hearing loss and causes a reduced amplification of sensory input that is associated with degraded frequency selectivity and wider auditory filters (Glasberg and Moore, 1986). As OHC loss is associated with elevated pure tone thresholds, this hearing deficit can routinely be quantified using the standard audiogram procedure (Johnson, 1970). However, especially those aspects of hearing-impairment which relate to sound

\footnotetext{
* Corresponding author. Department of Medical Physics and Acoustics, Carl von Ossietzky University of Oldenburg, Carl-von-Ossietzky-Straße 9-11, 26129, Oldenburg, Germany.

E-mail addresses: markus.garrett@uol.de (M. Garrett), s.verhulst@ugent.be (S. Verhulst).
}

perception deficits in people with normal hearing thresholds, are not sufficiently characterised by the audiogram alone (Kumar et al., 2007; Hind et al., 2011; Kobel et al., 2017; Lobarinas et al., 2017). These so-called suprathreshold hearing deficits, in the presence of normal sound detection, are a topic of intensive investigation as the underlying cause of these deficits may explain why two individuals with the same audiogram can have very different speech intelligibility scores (Festen and Plomp, 1983).

One possible cause for suprathreshold hearing deficits with otherwise normal hearing sensitivity is synaptopathy or cochlear neuropathy. Research in mice has shown that an overexposure to noise can lead to a loss of up to $50 \%$ of the synapses and cochlear nerve terminals innervating the inner hair cells (IHC) while hearing thresholds are normal (Kujawa and Liberman, 2009). Cochlear synaptopathy is hypothesised to be induced by glutamate excitotoxicity in the post-synaptic terminals of IHCs and the consequences are swelling, bursting and finally the withdrawal of the terminal dendrite (Liberman and Kujawa, 2017). Because each auditory nerve (AN) fibre communicates only with one IHC per tonotopic location along the basilar membrane (Stamataki et al., 
2006), even a partial deafferentation leads to a loss of information in the chain of information transfer that could eventually lead to neurodegeneration of the spiral ganglion cells (SGC; Liberman and Kujawa, 2017). Low-spontaneous rate (low-SR) fibres with high firing thresholds are relatively more affected by noise exposure (Furman et al., 2013) or aging (Schmiedt et al., 1996) than those with low thresholds and high-spontaneous firing rates (high-SR fibres). Given its expression, synaptopathy in low-SR fibres is thought to degrade the robust encoding of suprathreshold temporal envelopes (Bharadwaj et al., 2014), or results in the loss of spectral contrast important for speech-in-noise decoding (Carney, 2018).

In animal studies, it is possible to study and relate histological findings of synaptopathy directly to non-invasive subcortical electrophysiological measures such as the auditory brainstem response (ABR) and the envelope following response (EFR). The most prominent finding is the correlation between the ABR Wave-I amplitude at moderate-to-high sound levels and the number of intact IHC synapses (Kujawa and Liberman, 2009; Lin et al., 2011; Furman et al., 2013). EFRs to modulated pure tones with modulation rates around $0.7-1 \mathrm{kHz}$ can also be a robust and indirect measure for noise or ageing-induced synaptopathy (Shaheen et al., 2015; Parthasarathy and Kujawa, 2018). The relationship between synaptopathy and auditory evoked potentials has opened avenues for a non-invasive diagnosis of synaptopathy in humans and the study of how synaptopathy influences sound perception.

For example, a stronger reduction of EFR strength with decreasing stimulus modulation depth was found to go along with degraded ITD and AM detection as well as poorer selective attention in listeners with otherwise normal audiograms (Bharadwaj et al., 2015). Reduced perceptual temporal coding abilities may thus be diagnosed using a relative EFR slope metric, which is the slope of a line fit through the EFR strength as a function of modulation depth. In a second example, the ratio between the hair-cellgenerated summating potential (SP) and the AN-neuron generated action potential (AP or ABR Wave I) was also suggested as a marker of synaptopathy in humans. It predicted the poorer wordrecognition-in-noise performance of participants with higher doses of self-reported noise exposure (Liberman et al., 2016). Further studies have related the ABR Wave-I amplitude to the amount of life time noise exposure (Bramhall et al., 2017; Valderrama et al., 2018) and tinnitus (Schaette and McAlpine, 2011) in accordance with noise-induced synaptopathy observations in rodents (Kujawa and Liberman, 2009; Furman et al., 2013; Möhrle et al., 2016). Lastly, Mehraei et al. (2016) argued that increased Wave-V latency for increasing background noise levels may emphasise the contribution of low-SR fibres to the ABR resulting from their high firing thresholds and delayed onset responses (Bourien et al., 2014). Smaller than normal Wave-V latency shifts would hence predict a loss of low-SR fibres.

Even though human evoked potential studies can only provide indirect evidence of synaptopathy, post-mortem human temporal bone studies have demonstrated that synaptopathy also affects humans by reporting that noise overexposure or aging can reduce the number of synapses and AN-terminals innervating the IHCs before hearing thresholds are affected. SGC counts performed in specimens with normal populations of hair cells, predict a mean annual loss of up to 100 SGC (Makary et al., 2011; Wu et al., 2018). Work in macaques further suggests that mammals are more resilient to hair cell loss, but that their vulnerability to cochlear synaptopathy is similar to that of rodent models (Valero et al., 2017). Nevertheless, the degree to which synaptopathy and the use of subcortical measures for diagnostics are transferable to humans is still a topic of debate due to species-specific differences in the physiology of hearing (Plack et al., 2016; Hickox et al., 2017;
Prendergast et al., 2017). Furthermore, humans show increased variation in physiological measures compared to animals due to the heterogeneity in tissue-conductance, head size, noise exposure across the life span and different genetic factors (Mitchell et al., 1989; Trune et al., 1988; Plack et al., 2016; Liberman and Kujawa, 2017; Lobarinas et al., 2017; Yeend et al., 2017). Especially in the elderly, a relative mix of peripheral and cognitive factors plays a role in speech understanding and suprathreshold auditory temporal processing (Humes et al., 2010; Humes, 2013). This multitude of factors, combined with the fact that subcortical EEG measures are only indirect indicators of synaptopathy, reduce the translation from animal studies to precise diagnostic metrics for synaptopathy in humans.

Despite a range of positive findings, there are also studies which did not find evidence for a causal relationship between sound perception/noise exposure history and electrophysiological measures of synaptopathy in humans. For example, a study investigating how noise exposure history related to suprathreshold functional hearing and the ABR Wave-I amplitude did not find significant relations between the metrics. Following a similar approach, noise exposure history did not relate to the ABR Wave-I amplitude, or EFR measures, in young adults with and without tinnitus (Fulbright et al., 2017; Guest et al., 2017), or in 100 participants with normal audiometric thresholds (Prendergast et al., 2017). From the listed studies, we can either conclude that noiseinduced cochlear synaptopathy might not play an important role in young adults with normal audiometric hearing thresholds, or that the adopted electrophysiological measures are not sensitive enough to reveal subtle differences in neural fibre populations in this particular group (see Bramhall et al. (2019) for an overview).

The interpretation of subcortical EEG metrics in terms of synaptopathy is further complicated by the presence of other peripheral contributors to hearing loss, such as OHC deficits. Even though cochlear synaptopathy is more pronounced with advancing age (Sergeyenko et al., 2013; Fernandez et al., 2015; Parthasarathy and Kujawa, 2018), impaired audiograms are also common in the ageing population (ISO, 1990). Aging listeners with impaired audiograms are thus likely to suffer from both $\mathrm{OHC}$ deficits and synaptopathy, rendering the interpretation of electrophysiological metrics complicated as they can be affected by both deficits. The quantification and isolation of cochlear synaptopathy from other coexisting contributors of hearing loss is therefore still a major unsolved problem in hearing diagnostics (Plack et al., 2016; Verhulst et al., 2016; Hickox et al., 2017; Kobel et al., 2017).

As a first step to disentangle peripheral hearing deficits from electrophysiological metrics, we investigated whether existing ABR/EFR metrics and their relative combinations for synaptopathy diagnosis in a young $\mathrm{NH}(\mathrm{yNH})$ control group $(25.5 \pm 4.2$ years) follow the same trends for an older hearing impaired (oHI) group $(65.8 \pm 7.9$ years $)$ with high-frequency sloping audiograms. The latter group has $\mathrm{OHC}$ deficits as verified using the audiogram and distortion-product otoacoustic emission (DPOAE) thresholds and is expected to also suffer from synaptopathy on the basis of recent studies relating the normal ageing process to synaptopathy expression (Sergeyenko et al., 2013; Parthasarathy and Kujawa, 2018; Wu et al., 2018). We hypothesise that if synaptopathy drives the considered electrophysiological metrics, the oHI group should have equally poor or worse metrics than the worst performing yNH participants. However, if the results of the oHI participants do not follow the trends expected from synaptopathy, and show a relationship to hearing sensitivity differences within the older group, the considered metric is likely impacted by both OHC and synaptopathy and may consequently not be a robust marker of synaptopathy in listeners with impaired audiograms. This study investigates how markers of synaptopathy developed for $\mathrm{NH}$ 
listeners differ in two extreme participant groups: a young control group (yNH) and a representative clinical population with mild sensorineural hearing loss (oHI). The study outcomes can help restrain the large parameter space of potentially appropriate diagnostic metrics for synaptopathy to identify measures that can quantify synaptopathy in the presence of normal or abnormal OHC function.

\subsection{Considered $A B R / E F R$ metrics and expected outcomes}

We report individual differences in electrophysiological response behaviour for multiple stimulus parameters such as bandwidth, modulation frequency and depth as well as sound pressure level (SPL) to provide a comprehensive view of the applicability of subcortical EEG metrics in the two listener groups. We incorporate a relative metric design to account for interindividual differences and further reduce measurement uncertainty by combining different measures. In our analysis, we consider relationships of the metrics to objective physiological markers of peripheral hearing, age and other electrophysiological markers. We investigate the following hypotheses which are motivated by extending the $\mathrm{NH}$ synaptopathy results to an older $\mathrm{HI}$ group with high-frequency hearing loss and a suspected high degree of synaptopathy.

\section{1) Reduced EFR magnitudes:}

On the basis of animal-synaptopathy findings (Shaheen et al., 2015; Parthasarathy and Kujawa, 2018) and reduced temporal envelope coding ability (Bharadwaj et al., 2014) we expect that the EFR strength is reduced in the oHI group. Because biophysical EFRmodel predictions (Verhulst et al., 2018a,b) support the idea that OHC loss does not strongly influence the EFR metric, we predict that reduced EFR amplitudes in the oHI group predominantly reflect their individual degree of synaptopathy. In line with this, individual differences in oHI-EFRs should not relate to hearing threshold differences in this group.

\section{2) Steeper EFR slope metric:}

If the EFR strength reflects temporal coding ability of the brainstem, the EFR amplitude slope as a function of modulation depth reduction should be steeper in the oHI group (Bharadwaj et al., 2015). If this is not the case, the EFR slope metric might not exclusively be sensitive to synaptopathy and reflect a contribution of $\mathrm{OHC}$ deficits as well.

3) Lower ABR amplitudes for equal SPL stimuli at suprathreshold levels:

The ABR Wave I should be reduced in the oHI group if synaptopathy is driving this metric (e.g. Kujawa and Liberman, 2009). We also expect reduced amplitudes for the Wave $\mathrm{V}$ if central gain compensation does not play a role. Because central gain compensation (i.e., normal Wave $V$ in the presence of a reduced Wave I) was mostly observed in young, but not older, animals with synaptopathy (Möhrle et al., 2016), we expect that the ABR Wave-V amplitudes of our advanced-age oHI group should relate well to how synaptopathy affects the ABR Wave-I.

\section{4) Shallower ABR amplitude slopes:}

If synaptopathy is the main parameter affecting the $A B R$ amplitude growth as a function of stimulus level, we expect a shallower growth slope for the oHI group. However, a potential loss of cochlear compression due to OHC deficits is expected to yield steeper ABR amplitude slopes (Verhulst et al., 2016). OHC deficits are also associated with longer low-level ABR latencies (Lewis et al., 2015) and steeper ABR latency slopes (Gorga et al., 1985; Verhulst et al., 2016) based on relatively stronger contributions of the intact apical portion of the basilar membrane. If synaptopathy drives the ABR amplitude metric, we expect to see shallower ABR slopes that do not relate to the ABR latency slope which is expected to reflect $\mathrm{OHC}$ loss.

\section{5) Relationship between EFR and ABR metrics:}

If the positive ABR amplitude slope and negative EFR slope both represent aspects of synaptopathy, we expect a positive correlation between these metrics under the assumption that the neuronal population generating the EFR can be seen as a subgroup of the fibres responding to the click-ABR. Likewise, we expect to see a relationship between individual EFR magnitudes and the EFR slope as a function of stimulus modulation depth, and of the EFR magnitude with the ABR amplitude slope.

6) Age as a predictor of peripheral hearing deficit metrics:

Lastly, as age can be associated with both synaptopathy and $\mathrm{OHC}$ deficits (e.g. Makary et al., 2011; Sergeyenko et al., 2013; Parthasarathy and Kujawa, 2018; Wu et al., 2018) we expect to see a reduction in the response strength of the EFR and ABR metrics between our groups. By design, our study considers extreme age differences between the $\mathrm{yNH}$ and oHI group and these age differences may influence multiple aspects: e.g. sensorineural hearing damage (OHC loss and synaptopathy), changes in inhibitory neurotransmitter systems (Khouri et al., 2011; Richardson et al., 2013) or cognitive factors (Humes, 2013). The large heterogeneity of internal and external factors such as genetic predisposition or noise exposure over the course of a life time further complicate matters in humans compared to highly controlled animal studies (Parthasarathy et al., 2018). We therefore argue that the age variable will not be a good objective predictor of individual variation in the tested peripheral hearing metrics. This is in contrast to the between-group effects, which we do expect to see, given the overall mean age difference of 40 years between the groups.

\section{Materials and methods}

\subsection{Participants}

The study included 22 young normal-hearing (yNH) participants between 14 and $32 \mathrm{y} / 0\left(\mathrm{M}_{\mathrm{age}}=25.5\right.$ years; $\mathrm{SD}_{\mathrm{age}}=4.2,15$ females) and 23 older hearing-impaired participants (oHI) between 48 and $77 \mathrm{y} / \mathrm{o}\left(\mathrm{M}_{\mathrm{age}}=65.8\right.$ years; $\mathrm{SD}_{\mathrm{age}}=7.9,11$ females $)$. Only one of the considered oHI participants had a history of recent hearing aid use. All yNH participants had normal pure-tone audiogram thresholds ( $\leq 20 \mathrm{~dB} \mathrm{HL}$ ) and no more than $10 \mathrm{~dB} \mathrm{HL}$ at $4 \mathrm{kHz}$ as assessed with a clinical audiometer (Auritec AT900, Hamburg, Germany) at frequencies between 0.125 and $8 \mathrm{kHz}$. The oHI participants had high-frequency sloping audiograms with hearing thresholds $\geq 20 \mathrm{~dB} \mathrm{HL}$ (up to $45 \mathrm{~dB} \mathrm{HL}$ ) at $4 \mathrm{kHz}$ as shown in the red/ orange traces in Fig. 1. Given the evidence that synaptopathy sets in before OHC loss can be detected (Sergeyenko et al., 2013; Fernandez et al., 2015; Parthasarathy and Kujawa, 2018; Wu et al., 2018) the oHI group is expected to reflect a wide range of sensory as well as neural hearing loss profiles. To assess OHC integrity and cochlear function, audiometric thresholds were complemented with a $4-\mathrm{kHz}$ pure tone detection threshold using a two-alternative forcedchoice (AFC) adaptive tracking procedure and DPOAE thresholds 


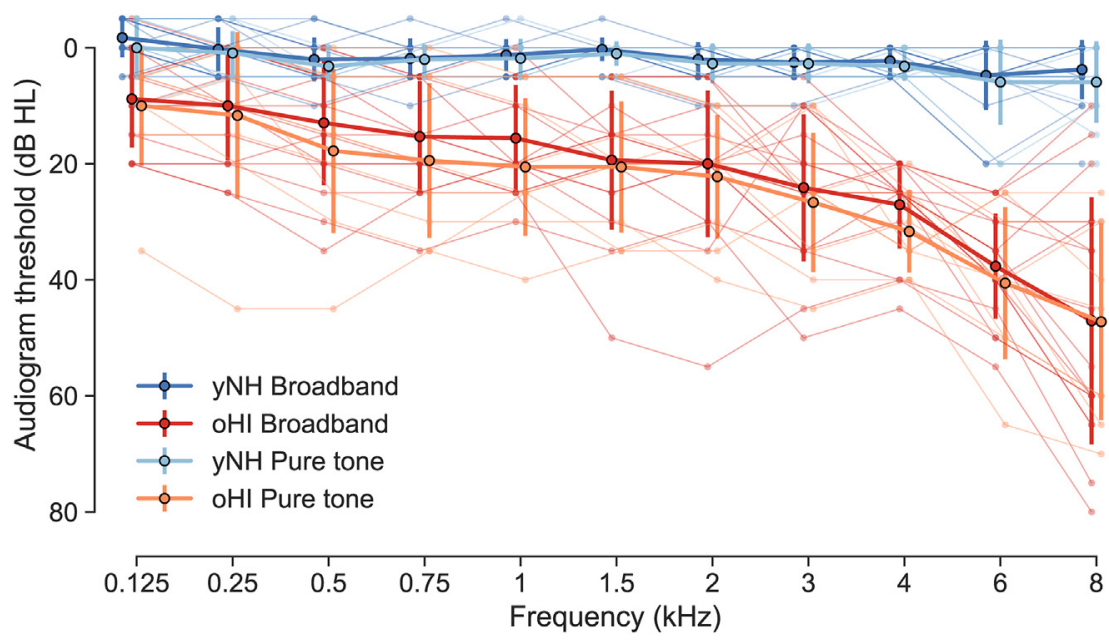

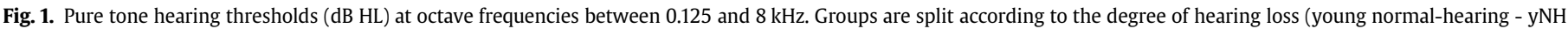

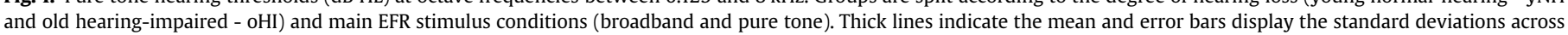
groups. The thin lines represent individual audiogram traces.

measured at $4 \mathrm{kHz}$. Two simultaneously presented pure tones with the primary frequencies $f_{1}$ and $f_{2}$ were exponentially swept up $(2 \mathrm{~s} /$ octave) at a fixed $f_{2} / f_{1}$ ratio of 1.2 over a $1 / 3$ octave range around the geometric mean of $4 \mathrm{kHz}$ using two ER2 (Etymotic Research, Elk Grove Village, IL, USA) speakers coupled to a ER10B + OAE microphone system (Etymotic Research). Stimulus presentation, recording and analysis was implemented using a custom-made MATLAB software-tool (Mauermann, 2013). Based on the primary level $\mathrm{L}_{2}$, the $\mathrm{L}_{1}$ levels were picked according to the Scissors paradigm (Kummer et al., 1998). Growth functions of the distortion component were computed as the average over 34 distortionsource DPOAE functions across the measured frequency range. Thresholds of the DPOAE were determined as the $\mathrm{L}_{2}$ level at which the extrapolated cubic fit curve reached a level of $-25 \mathrm{~dB}$ SPL (1.12 $\mu \mathrm{Pa}$; Boege and Janssen, 2002). Further details on the DPOAE threshold procedure can be found in Verhulst et al. (2016). Participants were informed about the experimental procedures according to the ethical guidelines at the University of Oldenburg. Written informed consent was obtained and participants were paid for their participation.

\subsection{Stimuli}

The amplitude-modulated EFR stimuli consisted of two main conditions. The broadband (BB) condition was designed to achieve a maximally broad basilar-membrane excitation while the pure tone (PT) was expected to maximise individual differences in the 4$\mathrm{kHz}$ range. Several other EFR conditions were collected from a subset of participants (see Table 1). The stimuli varied in bandwidth, SPL, modulation depth (MD) and modulation frequency $\left(\mathrm{f}_{\mathrm{m}}\right)$.

The BB stimuli consisted of a 75-dB-SPL white noise carrier that was amplitude-modulated with a modulation frequency of $120 \mathrm{~Hz}$.
The 4-kHz PT stimuli were calibrated to $70 \mathrm{~dB}$ SPL. For both stimulus types, three different modulation depths $0,-4,-8 \mathrm{~dB}$ (equivalent to 100,63 and $40 \%$ depth) were used, yielding a total of six main conditions. All other conditions were only tested at $100 \%$ modulation depth. The narrowband (NB) stimuli had a white noise carrier but were band-limited to one octave centred around $4 \mathrm{kHz}$. This stimulus was designed to achieve good signal strength while retaining frequency specificity around the centre frequency. The narrowband stimuli were tested at 75 and $70 \mathrm{~dB}$ SPL to allow for a comparison to the broadband and the pure tone data, and to assess the influence of SPL on the EFR. The 'broadband 480 ' condition only differed from the broadband condition by its modulation frequency of $480 \mathrm{~Hz}$. This higher modulation frequency was included to target more peripheral generators than the brainstem (Purcell et al., 2004) and might be more directly related to AN processing. Each broadband EFR stimulus lasted $600 \mathrm{~ms}$ followed by a uniformly distributed random silence jitter ( $>90$ and $<110 \mathrm{~ms}$; mean $=100 \mathrm{~ms}$ ). All narrowband and pure-tone stimuli were ramped using a $5 \%$ tapered-cosine window and were repeated 800 times, whereas all broadband stimuli were repeated only 600 times because they elicited more robust EFRs. Both polarities (50\% each) were presented. Due to time restrictions, not all participants were tested in all possible conditions. Nine yNH and three oHI participants took part in both main conditions (BB, PT), all other participants were only exposed to either the BB or PT condition.

Each ABR epoch had a duration of $30 \mathrm{~ms}$ and consisted of a $80-\mu \mathrm{s}$ condensation click followed by silence. After each epoch, a short uniformly distributed random silence jitter $(>0$ and $<3 \mathrm{~ms}$; mean $=1.5 \mathrm{~ms}$ ) was added. 7000 epochs were presented at a rate of $33.3 \mathrm{~Hz}$ for all four tested conditions: 70, 80, 90 and $100 \mathrm{~dB}$ peakequivalent sound pressure level (peSPL).

EEG recording took place in a double-walled electrically

Table 1

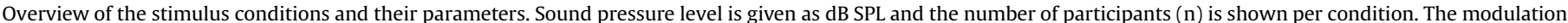
depth (MD) is in $\mathrm{dB}$ relative to $100 \%$ modulation.

\begin{tabular}{|c|c|c|c|c|}
\hline & Broadband $120 \mathrm{~Hz}$ & Pure tone $120 \mathrm{~Hz}$ & Narrowband $120 \mathrm{~Hz}$ & Broadband $480 \mathrm{~Hz}$ \\
\hline yNH & $\begin{array}{l}75 \mathrm{~dB} ; \mathrm{n}=20 \\
\mathrm{MD}=0,-4,-8\end{array}$ & $\begin{array}{l}70 \mathrm{~dB} ; \mathrm{n}=11 \\
\mathrm{MD}=0,-4,-8\end{array}$ & $\begin{array}{l}75 \mathrm{~dB} ; \mathrm{n}=20 ; \mathrm{MD}=0 \\
70 \mathrm{~dB} ; \mathrm{n}=11 ; \mathrm{MD}=0\end{array}$ & $75 \mathrm{~dB} ; \mathrm{n}=20 ; \mathrm{MD}=0$ \\
\hline $\mathrm{oHI}$ & $\begin{array}{l}75 \mathrm{~dB} ; \mathrm{n}=17 \\
\mathrm{MD}=0,-4,-8\end{array}$ & $\begin{array}{l}70 \mathrm{~dB} ; \mathrm{n}=9 \\
\mathrm{MD}=0,-4,-8\end{array}$ & $\begin{array}{l}75 \mathrm{~dB} ; \mathrm{n}=14 ; \mathrm{MD}=0 \\
70 \mathrm{~dB} ; \mathrm{n}=9 ; \mathrm{MD}=0\end{array}$ & $75 \mathrm{~dB} ; \mathrm{n}=20 ; \mathrm{MD}=0$ \\
\hline
\end{tabular}


shielded measurement booth. Participants sat comfortably in a reclining chair while watching a silent movie. All stimuli were presented monaurally (better ear based on the audiogram) using foam tips coupled to magnetically-shielded ER-2 insert earphones (Etymotic Research) which were connected to a TDT-HB7 headphone driver (Tucker-Davis, Alachua, FL, USA) and a Fireface UCX sound card (RME, Haimhausen, Germany). All stimuli were generated in MATLAB at a sampling rate of $48 \mathrm{kHz}$ and calibrated using an oscilloscope (for ABR only), B\&K type 4157 ear simulator and sound level meter type 2610 (Brüel \& Kjær, Nærum, Denmark). EEGs were recorded using a 32-channel EEG cap and amplifier (Biosemi, Amsterdam, Netherlands) with a sampling rate of $16384 \mathrm{~Hz}$ and 24bit $\mathrm{AD}$ conversion. Common-mode-sense and driven-right-leg electrodes (CMS/DRL) were placed near the vertex of the participant. The data were re-referenced to the offline averaged earlobe electrodes. Only the vertex electrode $(\mathrm{Cz})$, yielding the overall best signal strength, was used for all further analyses to mimic recording conditions found in clinical settings. Electrode offsets (DC values of the common mode signal) were kept below $20 \mathrm{mV}$.

\subsection{Data processing and analysis}

All EFR data were pre-processed using the Python programming language (version 2.7.10 | Anaconda 2.3.0 (64-bit), www.python. org) and MNE-Python (version 0.9.0) (Gramfort et al., 2013, 2014). An EOG channel was constructed from the electrodes Fp1 and Fp2 to determine the weights for the independent component analysis (ICA) eye movement artifact detection. To this end, the data were filtered $(1-40 \mathrm{~Hz})$ using a 4 th order infinite impulse response (IIR) Butterworth filter and were epoched to $1 \mathrm{~s}$ long chunks using an epoch rejection threshold of $150 \mu \mathrm{V}$. The MNE implemented fast ICA algorithm was applied (max. 300 iterations) and muscle artifacts were determined (Debener et al., 2010). The ICA weights were then applied to the original unfiltered data to remove artifacts. On average two ICA components relating to lateral eye movement and blinks were removed.

\subsubsection{Envelope following response}

The eye-artifact-free data were high-pass filtered at $60 \mathrm{~Hz}$ and then low-pass filtered at $650 \mathrm{~Hz}$ using a 4th order IIR Butterworth filter. A zero-phase shift was achieved by applying a forwardbackward filter procedure. Data were epoched from $-0.01-0.6 \mathrm{~s}$ around trigger onset. The $10-\mathrm{ms}$ trace before trigger onset was used for baseline correction and discarded afterwards. Epochs with amplitudes exceeding a $100 \mu \mathrm{V}$ threshold were removed. Each epoch was transformed to the frequency domain using Matlab's Fast Fourier Transform (FFT) function. To estimate the frequency dependent noise floor, a bootstrap procedure was applied (Schimmel, 1967; Zhu et al., 2013). First, a magnitude spectrum estimate of the neural responses for each condition and participant was computed by averaging randomly drawn epochs. The random draws (with replacement) equalled the number of epochs left after artifact rejection in each condition. This step was repeated 200 times resulting in an estimated magnitude spectrum distribution. The average spectrum of this approximately Gaussian distributed measure was used as the estimate of the individual participant's magnitude spectrum of the response per condition. The standard deviation of the 200 estimates was used as an estimator of the variability. The spectral magnitude of the noise floor was calculated using a similar approach, except for that the number of frequency estimates was increased to 1000 and that the phase of half of the randomly drawn epochs was flipped. This method cancels out the constant time-locked signal (i.e., the EFR) in the recording and only preserves the non-stationary noise that has a characteristic shape proportional to $1 / \mathrm{f}$ (Voytek et al., 2015). The estimated noise floor and the response estimate were then transformed to a $\mathrm{dB}$ scale using $20 * \log _{10}($ signal $/ \sqrt{2}$ ). The reference $\sqrt{2}$ (Bharadwaj et al., 2015) reflects the amplitude of a pure tone with a root-meansquare value of one. Lastly, the estimated noise floor was subtracted from the EFR magnitude to yield a signal-to-noise ratio measure (EFR SNR) which was used in all further EFR analyses and is referred to as EFR or EFR magnitude. Responses were considered as significantly above the statistical noise floor, hence showing a high reliability, if their magnitude at the modulation frequency exceeded the 1000 computed noise floor estimates in more than $95 \%$ of all cases. The EFR normalisation to the noise floor allows for a better comparison of EFR SNRs between individuals as it takes into account individual differences in background noise floor level. The EFR normalisation is also particularly important when comparing EFRs across different modulation frequencies as both the modulation transfer function (Purcell et al., 2004; Tichko and Skoe, 2017) and the background noise levels are frequency dependent.

\subsubsection{Auditory brainstem response}

The ABR data were band-pass filtered between 200 and $1500 \mathrm{~Hz}$ using a 4th order Butterworth filter and a forward-backward procedure to avoid phase shifts. Data were epoched between -5 and $20 \mathrm{~ms}$ around the stimulus onset, baseline corrected ( -5 to $0 \mathrm{~ms}$ ) and a threshold criterion of $40 \mu \mathrm{V}$ was applied for epoch rejection. The ABR waveforms were estimated using a bootstrap procedure similar to that adopted for the EFR data. For each participant, 200 wave estimates were computed using 6000 randomly picked epochs per estimate. The average of the 200 waveforms was used as the $A B R$ response. ABR latencies and peak amplitudes of Wave I and $\mathrm{V}$ were extracted by visual inspection using customised MATLAB scripts. Only ABR data points that exceeded the 1000 computed ABR noise floor estimates at the extracted latency of Wave I and V in 95\% of all cases (as described for the EFRs) were considered to lie above the statistical noise floor. All reported latencies were compensated for by the fixed recording delay of the sound delivery system $(1.16 \mathrm{~ms})$.

\subsubsection{Statistical analysis}

The assumptions for the performed statistical inference tests were tested using the 'SciPy' python package for scientific computing (Oliphant, 2007; Millman and Aivazis, 2011). Normal data distribution was tested using the Shapiro-Wilk-Test and the equal-variance assumption was assessed using the Levene-Test. If assumptions were satisfied, dependent/independent t-tests were used to test differences between two samples. If the normaldistribution assumption was not met for two independent samples, the non-parametric Mann-Whitney $U$ Test (U) was applied. If only the equal-variance assumption was violated, Welch's $t$-test was performed. If two dependent samples violated the normaldistribution assumption, the Wilcoxen signed-rank test (W) was applied. All correlations reported refer to the Pearson correlation coefficient ( $r$ ) if both variables were normally-distributed, otherwise Spearman's rank correlation coefficient $(\rho)$ was used.

All analyses of variance (ANOVA) and post-hoc analyses were done in the R programming environment (R Core Team, 2017) using the 'nlme' (Pinheiro et al., 2017) and 'lsmeans' (Russell, 2016) packages. All reported p-values for multiple comparisons were Bonferroni adjusted to control for the family-wise error rate and can be directly compared to the applied significance level of $\alpha=0.05$.

\section{Results}

To compare the $\mathrm{OHC}$ integrity between the two groups, an independent $t$-test for the $4-\mathrm{kHz}$ DPOAE thresholds was applied. The 
oHI group $(28.39 \pm 11.00 \mathrm{~dB}$ SPL) showed significantly higher thresholds $(\mathrm{t}(42)=-6.11 ; \mathrm{p}<0.0001)$ compared to the $\mathrm{yNH}$ group $(8.42 \pm 10.13 \mathrm{~dB}$ SPL). DPOAE thresholds were significantly correlated to the 4-kHz audiometric thresholds $(\rho=0.58 ; \mathrm{p}=0.0001$; $\mathrm{N}=40$ ) and the 4-kHz AFC thresholds ( $\rho=0.86 ; \mathrm{p}<0.0001$; $\mathrm{N}=30$ ) in agreement with a large population study (Boege and Janssen, 2002). The 4-kHz AFC and audiometric thresholds also showed a strong correlation $(\rho=0.91 ; \mathrm{p}<0.0001 ; \mathrm{N}=32)$.

The EFR magnitudes for the main BB and PT stimulus conditions are illustrated in Fig. 2 for the different participant groups and three modulation depths. All data points show a positive SNR but data points that lie below the statistical noise floor are indicated with square markers. A two-factor $(2 \times 3)$ mixed design ANOVA comparing all presented data points for the BB-EFR magnitudes indicated a significant main effect of group $(F(1,35)=57.88$; $\mathrm{p}<0.0001)$ and modulation depth $(\mathrm{F}(2,70)=61.60 ; \mathrm{p}<0.0001)$ but no significant interaction. A post-hoc analysis of pairwise comparisons (15 comparisons) showed significantly lower EFR magnitudes for the oHI participants in all three modulation depth conditions compared to the yNH group. As expected, decreasing the modulation depth resulted in smaller EFRs. EFR magnitudes were significantly smaller between the $0 \mathrm{~dB}$ and $-8 \mathrm{~dB}$ conditions as well as for the $-4 \mathrm{~dB}$ and $-8 \mathrm{~dB}$ contrast in both groups. No significant differences were observed between the $0 \mathrm{~dB}$ and $-4 \mathrm{~dB}$ conditions in either of the groups. The PT-EFRs showed similar outcomes, with a significant main effect of group $(F(1,18)=26.34 ; p<0.0001)$ and modulation depth $(\mathrm{F}(2,36)=6.08 ; \mathrm{p}=0.0053)$. The interaction did not reach significance. The post-hoc tests (15 comparisons) only showed significant differences between the groups for the 0 and -4 $\mathrm{dB}$ modulation depth conditions, and between the 0 and $-8 \mathrm{~dB}$ conditions within the yNH group. When only considering the EFRs that exceeded the statistical noise floor in the analysis (see methods), the reduction of EFR magnitudes with decreasing modulation depth was strongly diminished for the PT and BB conditions in the oHI group and for the PT condition in the yNH group. The percentage of participants with EFR magnitudes above the statistical noise floor is shown in Table 2 for the BB and PT conditions.

The increased number of EFRs lying below the statistical noise floor as the modulation depth decreased was especially prominent for oHI participants, underlining the increased difficulty for the brainstem to encode envelope information robustly in these listeners. The influence of the carrier type (BB vs. PT) was investigated using a two-factor $(2 \times 3)$ mixed design ANOVA. The carrier type
Table 2

Percentage of participants per carrier (BB,PT), group (yNH/oHI) and modulation depth condition $(0,-4,-8 \mathrm{~dB}$ re: $100 \%)$ whose raw estimated EFR response at the modulation frequency $(120 \mathrm{~Hz})$ exceeded the 1000 individually estimated noise floor values more than $95 \%$ of the time.

\begin{tabular}{llll}
\hline & MD: 0 dB & MD: 4 dB & MD: 8dB \\
\hline yNH Broadband & $100 \%$ & $100 \%$ & $95.0 \%$ \\
oHI Broadband & $82.4 \%$ & $70.6 \%$ & $29.4 \%$ \\
yNH Pure tone & $100 \%$ & $81.8 \%$ & $63.6 \%$ \\
oHI Pure tone & $33.3 \%$ & $11.1 \%$ & $11.1 \%$ \\
\hline
\end{tabular}

showed a significant main effect for the yNH $(F(1,29)=19.60$; $\mathrm{p}=0.0001)$ and oHI participants $(\mathrm{F}(1,24)=10.56 ; \mathrm{p}=0.0034)$. The $\mathrm{BB}$ carrier resulted in higher EFR values, as confirmed through posthoc analysis (15 comparisons) for all MD conditions in the $\mathrm{yNH}$ group $(0.0079 \leq \mathrm{p} \leq 0.0403)$ and for the 0 and $-4 \mathrm{~dB}$ conditions $(0.0207 \leq \mathrm{p} \leq 0.0477)$ in the oHI group.

To reduce the potential influence of confounding factors such as head size, skull thickness and gender on the EFR magnitudes, a relative self-normalising slope metric to quantify suprathreshold coding fidelity was used (Bharadwaj et al., 2014). The data points $(\mathrm{m} \approx 1,0.63,0.4)$ corresponding to $20 * \log _{10}(\mathrm{~m})=0,-4,-8 \mathrm{~dB}$ modulation depth were used to fit a straight line through the EFR magnitudes as a function of the stimulus modulation depth. All data points, including those below the statistical noise floor (but with positive SNRs), were used in the slope calculation. EFR slopes are shown in Fig. 3 and the yNH slopes corroborate those reported in other studies (Bharadwaj et al., 2015; Guest et al., 2017). The PTEFR slopes showed a greater variance than the BB-EFR slopes in the $\mathrm{yNH}$ group and independent two-sided t-tests comparing $\mathrm{yNH}$ and oHI participants in the $\mathrm{BB}(\mathrm{t}(35)=-2.02 ; \mathrm{p}=0.0510)$ and PT conditions ( $\mathrm{t}(18)=-1.63 ; \mathrm{p}=0.1209)$ did not indicate significant differences. This result was unexpected given that shallower EFR slopes are associated with better temporal envelope coding ability (Bharadwaj et al., 2015). As the oHI group is expected to suffer from synaptopathy, their EFR slopes were expected to be steeper than normal. Opposite to what was expected from the synaptopathyhypothesis, oHI participants had shallower mean slopes than the yNH group, and some participants even showed positive slopes. To better understand the observed slopes, we investigated how the EFR slopes were related to the EFR magnitudes (see Fig. 4). The yNH-EFR slopes only showed a significant correlation to the $-8-\mathrm{dB}$
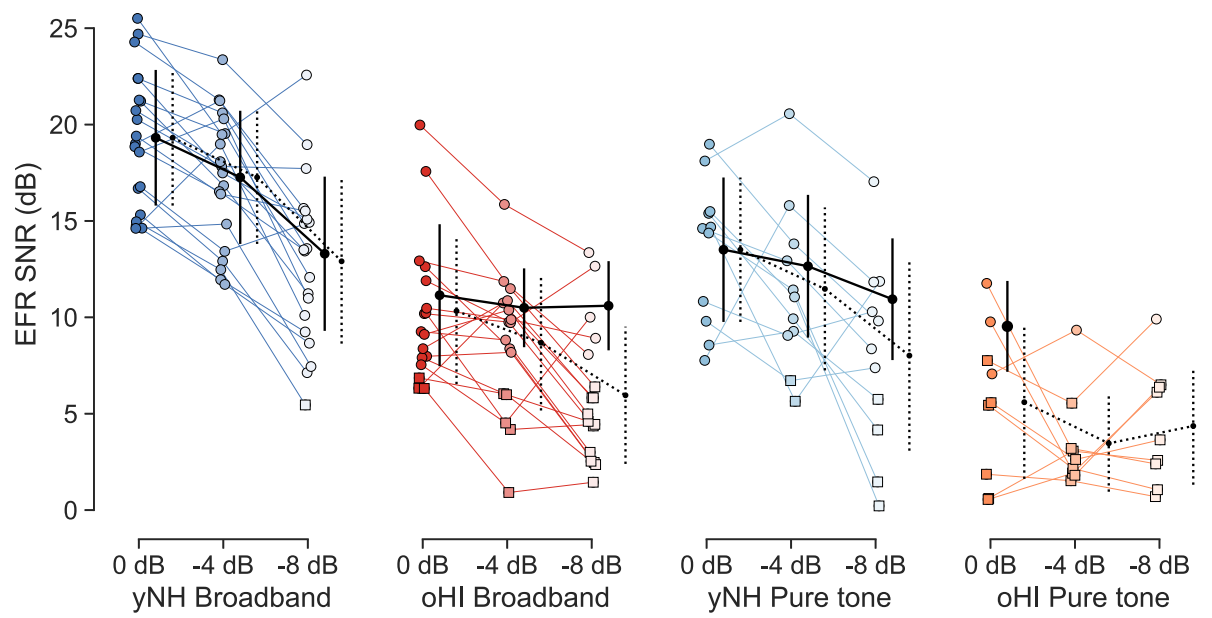

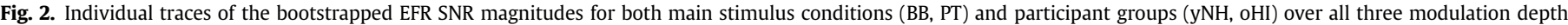

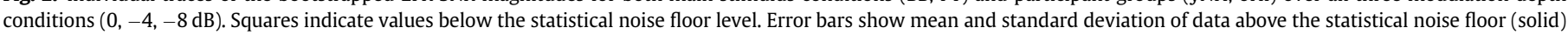
and all data (dashed) across groups. 


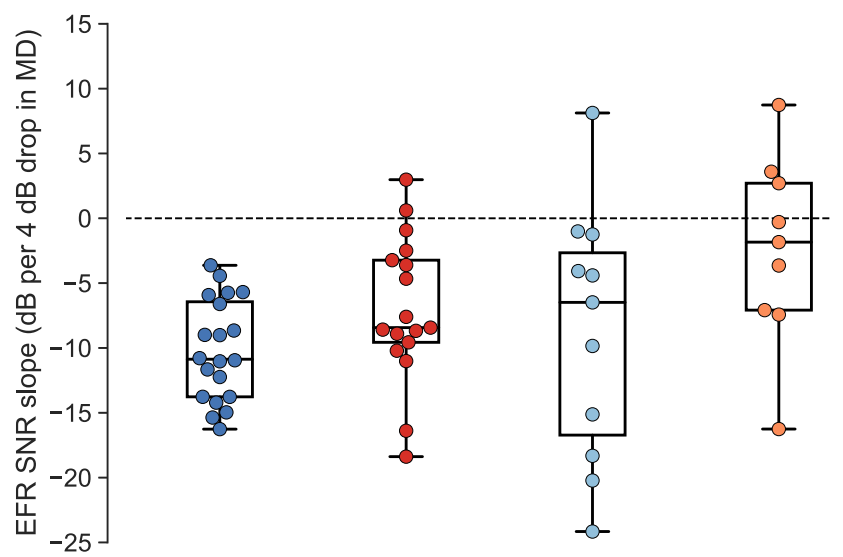

yNH Broadband oHI Broadband yNH Pure tone oHI Pure tone

Fig. 3. Boxplots and individual data points of the EFR SNR slopes (slope of a straight line fitted through all three modulation depth conditions) for both stimulus conditions (BB, PT) and groups (yNH, oHI). More negative values indicate a steeper EFR SNR reduction with decreasing modulation depth.

MD EFR condition (BB: $r=-0.51, p=0.0215 ; \quad P T: r=-0.76$, $\mathrm{p}=0.0062$ ) indicating that the EFR slope was mostly determined by the degree of temporal coding at lower modulation depths. In contrast, the oHI-EFR slopes correlated strongest with the 0-dB MD EFR condition (BB: $\rho=0.47, p=0.0551$; PT: $r=0.76, p=0.0169$ ). Their slope was therefore mainly determined by the degree of temporal coding at higher modulation depths.

With one exception, we did not find significant correlations between the EFR magnitudes in the different conditions and the 4$\mathrm{kHz}$ audiogram or $4-\mathrm{kHz}$ DPOAE thresholds within the $\mathrm{yNH}$ or oHI subgroups. Given that synaptopathy does not affect hearing thresholds (Kujawa and Liberman, 2009), these results fall within the expectation that suprathreshold temporal envelope coding is independent of hearing sensitivity. However, at $4 \mathrm{kHz}$, we did see a single positive correlation between the PT-EFR (100\% MD) and the audiogram threshold within the oHI group $(r=0.68, p=0.0430$, $\mathrm{N}=9$ ). Of all oHI participants, the largest EFR magnitudes were found for individuals with audiogram thresholds $>30 \mathrm{~dB}$ HL (i.e. greater hearing loss). This result might be explained by a mechanism in which greater degrees of $\mathrm{OHC}$ loss result in a linearisation of cochlear processing, which could counteract the EFR reduction associated with synaptopathy. EFR magnitudes above the statistical noise floor were recorded for the majority of oHI participants for $100 \%$ modulated stimuli, suggesting that the EFR metric itself might be robust for usage in listeners with sloping high-frequency audiograms. However, as the PT-EFRs were only significant above the statistical noise floor for individuals with thresholds $>30 \mathrm{~dB} H \mathrm{HL}$, OHC deficits might interact with synaptopathy to affect the EFR magnitude (at least in the PT condition). The only significant link between the hearing sensitivity measures (4-kHz audiogram/ DPOAE thresholds) and the EFR slope metric was found for the 4$\mathrm{kHz}$ audiogram thresholds in the oHI-PT group $(\mathrm{r}=0.86$, $\mathrm{p}=0.0032, \mathrm{~N}=9$ ) which can be explained by how the slope relates to the EFR magnitudes (see above).

\subsection{Effect of bandwidth and sound pressure level}

A stimulus bandwidth change from BB to $\mathrm{NB}(\mathrm{t}(19)=5.48$; $\mathrm{p}<0.0001)$ and again from NB to PT $(\mathrm{t}(10)=4.76 ; \mathrm{p}=0.0009)$ reduced the yNH-EFRs significantly (left panel of Fig. 5A). A significant effect of bandwidth on the oHI-EFRs (right panel of Fig. 5A) was not found. The 5-dB stimulus level difference between the NB conditions reached significance $(t(29)=-2.05 ; \mathrm{p}=0.0498)$ for the yNH group, showing a larger EFR mean for the 70-dB-SPL than for the 75-dB-SPL NB condition. This level effect was still present when only including participants who participated in both conditions $(\mathrm{t}(8)=-2.53, \mathrm{p}=0.0354)$. For the oHI participants, this difference only reached significance on a group level when considering all data points $(\mathrm{t}(21)=2.60, \mathrm{p}=0.0168)$. The oHI participants showed significantly smaller EFRs than the yNH participants in both NB conditions (75 dB: $\mathrm{t}(32)=4.34 ; \mathrm{p}=0.0001$ and $70 \mathrm{~dB}: \mathrm{t}(18)=7.35$; $\mathrm{p}<0.0001)$.

\subsection{Effect of modulation frequency}

When comparing EFR magnitudes to the modulation frequencies of 120 and $480 \mathrm{~Hz}$ (Fig. 5B), the yNH participants showed a significant decrease with increasing modulation frequency $(t(19)=6.98 ; p<0.0001)$, even when only considering responses above the statistical noise floor $(\mathrm{t}(13)=5.80 ; \mathrm{p}=0.0001)$. The correlation between the two EFR conditions showed a positive trend but did not reach significance $(\mathrm{r}=0.35 ; \mathrm{p}=0.13, \mathrm{~N}=20)$. For oHI participants, where the majority of $480-\mathrm{Hz}$ EFRs were below the statistical noise floor level, the modulation frequency increase also reduced the EFR magnitude significantly $(\mathrm{W}=7.0 ; \mathrm{p}=0.001)$. An independent $t$-test between groups for the $480-\mathrm{Hz}$ conditions revealed that $\mathrm{yNH}$ participants had significantly larger EFRs

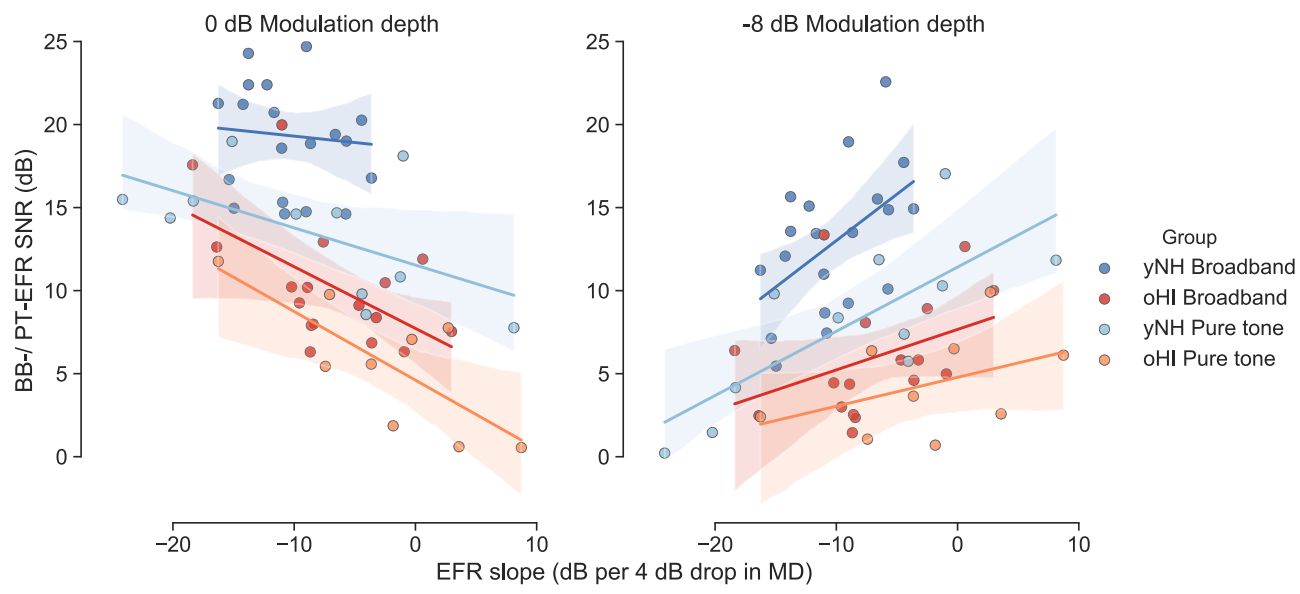

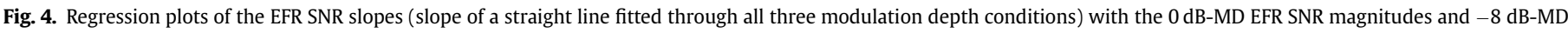
EFR SNR magnitudes for both main stimulus conditions (BB, PT) and participant groups (yNH, oHI). The shaded areas display the $95 \%$ confidence interval of the regression fit. 

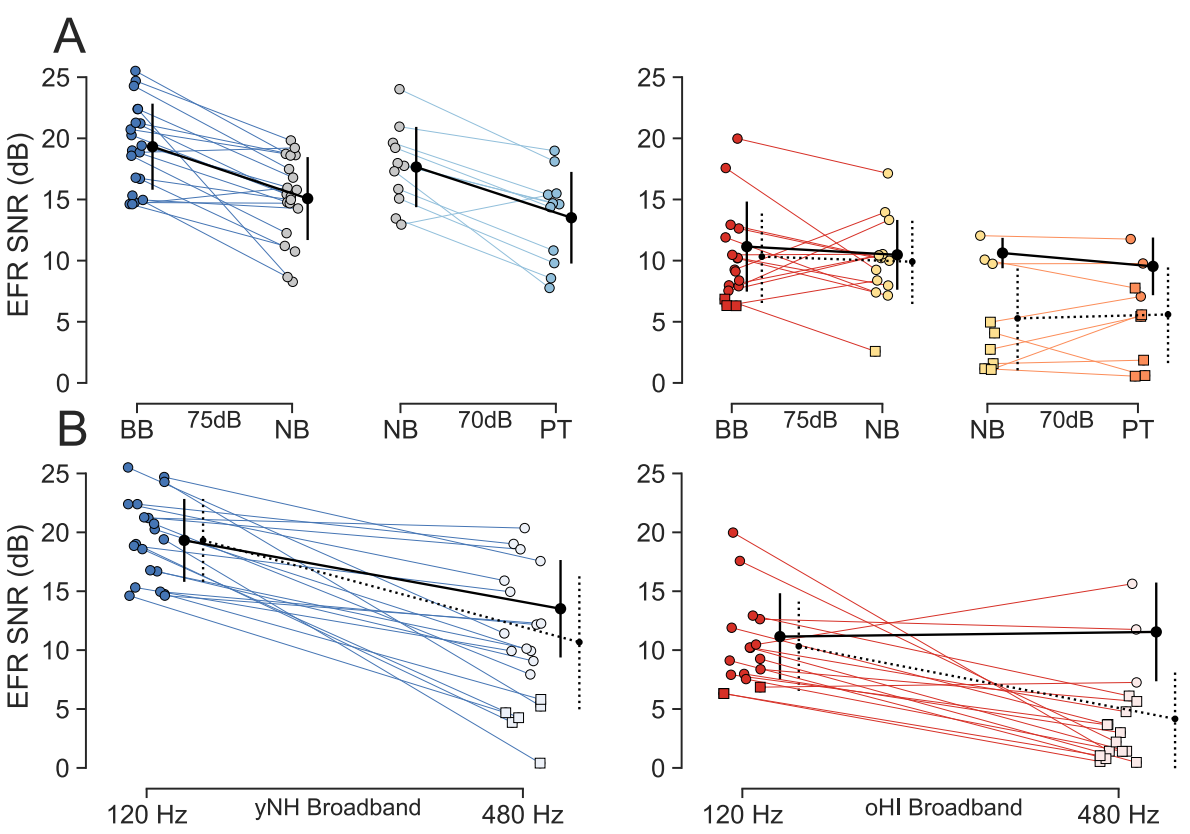

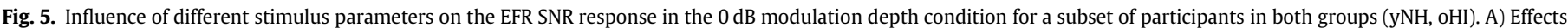

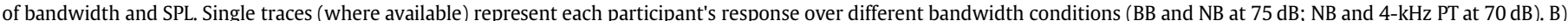

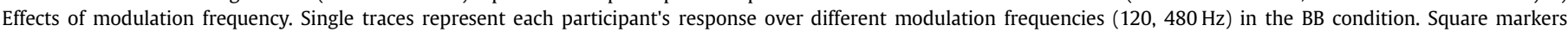

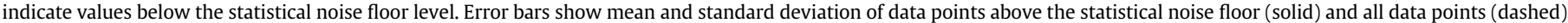
across participants in a group.

$(U=59.0, p=0.0004)$ than the oHI group.

\subsection{Auditory brainstem responses}

The ABR traces were analysed for Wave I and V, and their respective amplitudes and latencies are shown in Fig. 6 . The insets show grand-average traces of the yNH (blue) and oHI (red) participants for all four level conditions. The different peSPL conditions are indicated by different shapes and colours (see colour bar).
Visually detectable peaks that were below the statistical noise floor are marked by white symbols. Due to the low number of significant Wave-I data points for the oHI group, statistical differences were only investigated for the yNH group using a mixed-design ANOVA. The results indicated a significant effect of peSPL on the Wave-I latency $(F(3,29)=78.18 ; p<0.0001)$ but not on the amplitude $(\mathrm{F}(3,29)=1.34 ; \mathrm{p}=0.2815)$. A post-hoc analysis (6 comparisons) indicated a significant latency reduction between all peSPL conditions, except for the 90-vs.-100 dB peSPL comparison.
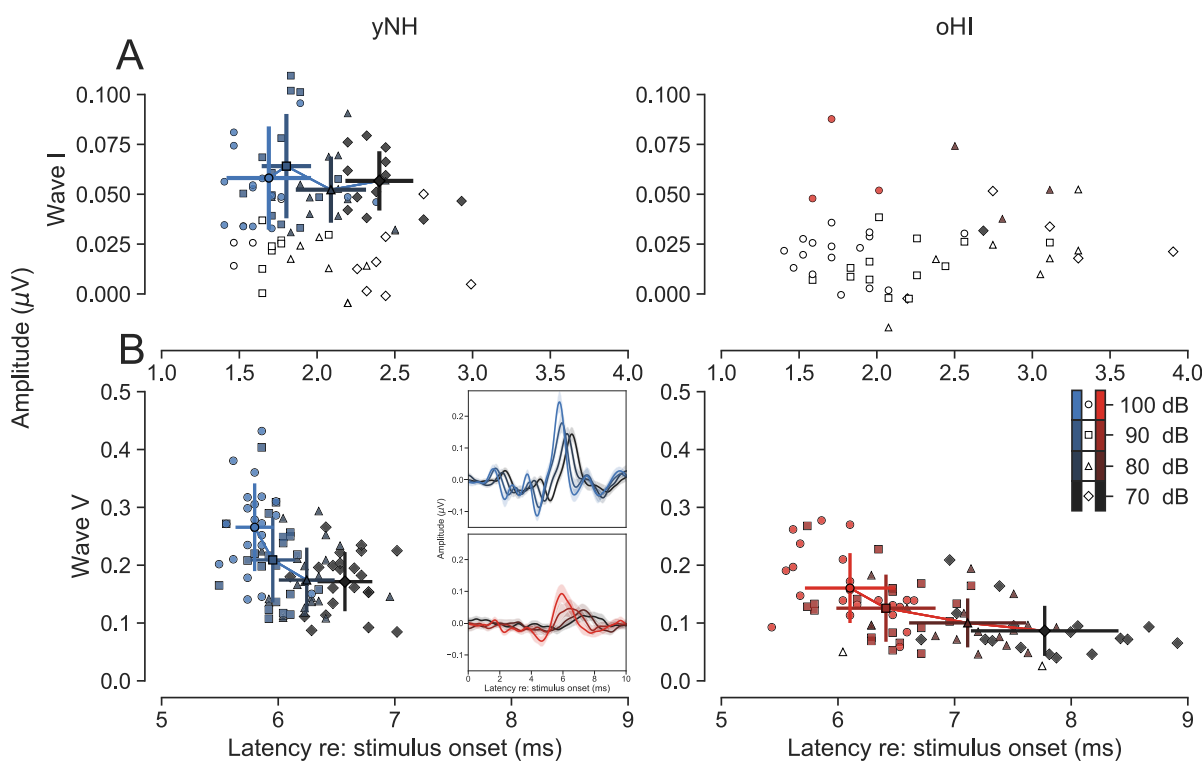

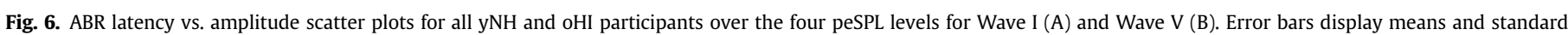

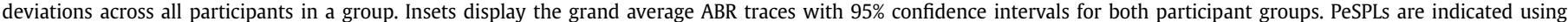

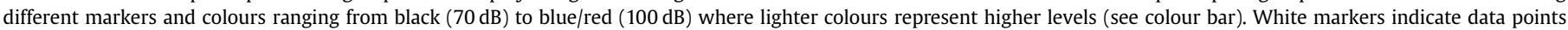
below the statistical noise floor. (For interpretation of the references to colour in this figure legend, the reader is referred to the Web version of this article.) 
The analysis for Wave $\mathrm{V}$ showed more reliable results for both groups. A two-factor mixed-design ANOVA for the Wave-V amplitudes indicated significant main effects of group $(F(1,42)=29.00$; $\mathrm{p}<0.0001)$ and peSPL $(\mathrm{F}(3,113)=69.42 ; \mathrm{p}<0.0001)$. The $\mathrm{yNH}$ participants showed larger amplitudes than the oHI participants in all four peSPL conditions $(0.0001<\mathrm{p} \leq 0.0014)$ as revealed by a post-hoc analysis (28 comparisons). The data are depicted in Fig. 7A where squares indicate data points below the statistical noise floor. Wave-V amplitudes increased with increasing peSPL in both groups and post-hoc pairwise comparisons (28 comparisons) confirmed this for most conditions. Exceptions that did not reach significance were the 70-vs.-80 dB-peSPL contrast in the yNH group, and the 70vs.-80 and 80 -vs.-90 dB-peSPL contrast in oHI participants. No significant interaction between group and peSPL was found. The Wave-V latencies were significantly shorter in yNH participants $(\mathrm{F}(1,42)=37.49 ; \mathrm{p}<0.0001)$, except for the $100 \mathrm{~dB}$-peSPL condition ( $\mathrm{t}$-ratio $=2.79, \mathrm{p}=0.22$ ). The latencies in both groups converged to each other for higher peSPLs (see panel 7B). Only for the $100 \mathrm{~dB}$ peSPL condition and for the oHI participants, did ABR amplitudes and latencies show a significant correlation $(\mathrm{r}=-0.50, \mathrm{p}=0.0060$, $\mathrm{N}=22$ ). The peSPL also showed a significant main effect ( $\mathrm{F}(3$, $113)=337.58 ; \mathrm{p}<0.0001$ ). Wave-V latencies decreased systematically with increasing peSPL in both groups and post-hoc pairwise comparisons (28 comparisons) for all but the yNH 90-vs.-100 dBpeSPL condition ( $\mathrm{t}$-ratio $=-2.86, \mathrm{p}=0.13$ ) reached significance, corroborating earlier reports (Lewis et al., 2015). All contrasts for the oHI participants reached significance, indicating a stronger influence of peSPL on the oHI latencies at higher peSPLs compared to the yNH participants, which is supported by a significant interaction effect $(F(3,113)=51.00 ; p<0.0001)$. Significant correlations of the ABR Wave-V amplitude (80 dB: $\rho=-0.48 ; \mathrm{p}=0.0490, \mathrm{~N}=17$ ) and the Wave-V latency ( $70 \mathrm{~dB}: \rho=0.54, \mathrm{p}=0.0249, \mathrm{~N}=17 ; 80 \mathrm{~dB}$ : $\rho=0.58, p=0.0148, N=17 ; 90 \mathrm{~dB}: \rho=0.48, \mathrm{p}=0.0360, \mathrm{~N}=19$ ) with the 4-kHz audiometric threshold were only observed in the oHI group. Smaller Wave-V amplitudes and longer latencies were associated with higher audiometric thresholds.

Similar to the EFR data, relative slope measures were computed from the Wave-V ABR data to reduce the effects of confounding factors on the absolute values. Such relative changes in ABR characteristics have successfully been linked to cochlear synaptopathy in the past (Mehraei et al., 2016). A straight line was fitted for each participant with a complete set of Wave-V peaks above the statistical noise floor through the four data points representing either the amplitude or latency information. Fig. 8 depicts the resulting

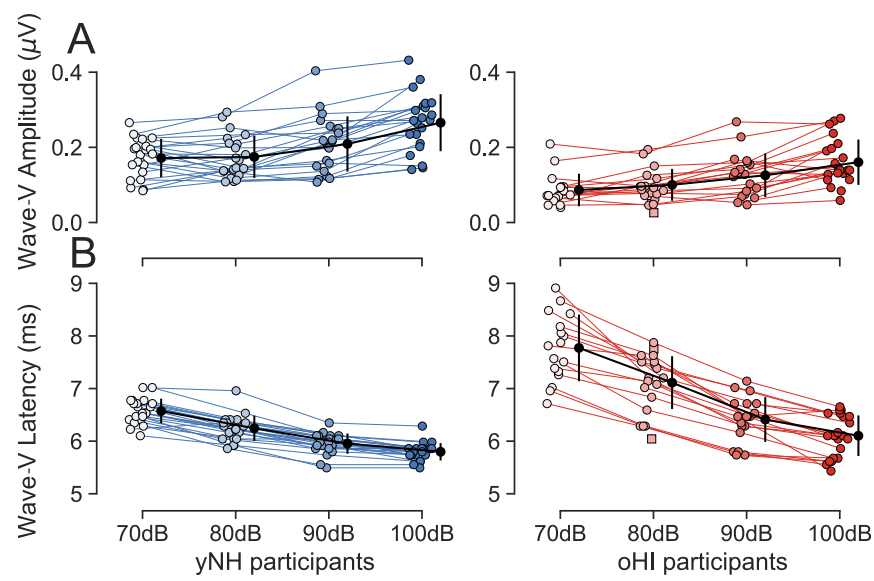

Fig. 7. ABR Wave-V amplitude (A) and latency traces (B) for all yNH and oHI participants over the four increasing sound pressure levels (peSPLs). Error bars display mean values and standard deviation in a group per peSPL condition. Square markers indicate data points below the statistical noise floor.
ABR amplitude (panel A) and ABR latency slope values (panel B) as a function of increasing peSPL for both groups. The yNH participants showed significantly shallower positive amplitude slopes $(t(35)=-3.69 ; \mathrm{p}=0.0008)$. Due to the violation of the equal variance assumption, Welch's $t$-test for independent samples was used to assess the latency slope differences between groups. The $\mathrm{yNH}$ group also showed shallower negative latency slopes $(t(17.21)=8.28 ; \mathrm{p}<0.0001)$ than the oHI group. The steeper oHI amplitude slopes did not follow the trend expected from synaptopathy which predicts shallower amplitude growth for participants with synaptopathy (Furman et al., 2013). The observed steeper negative oHI latency slopes fell in line with how sloping highfrequency audiograms are expected to affect the ABR latency slope (Gorga et al., 1985). This is further supported by the significant link between the ABR latency slope and the $4-\mathrm{kHz}$ audiometric threshold as a measure of hearing sensitivity $(\rho=-0.522$; $\mathrm{p}=0.0458 ; \mathrm{N}=15$ ) found in the oHI participant group. Steeper ABR Wave-V slopes were hence associated with higher audiometric thresholds.

Lastly, the ratio between the ABR Wave-I and Wave- $\mathrm{V}$ amplitudes was computed for the 100-dB peSPL condition as a selfnormalising measure thought to reflect central gain in the auditory brainstem (Schaette and McAlpine, 2011; Möhrle et al., 2016; Valderrama et al., 2018). Due to the low number of Wave-I peaks above the statistical noise floor in the oHI group, no statistical comparisons were made between the groups. The Wave-I/V ratios for both groups are depicted in Fig. 9. White data points represent ratios based on one or both wave peaks below the statistical noise floor.

\subsection{Relationship between ABR and EFR metrics}

To investigate relationships between different potential electrophysiological measures of synaptopathy, a correlation analysis was performed for the most reliable EFR condition (100\% MD) at $120 / 480 \mathrm{~Hz}$ and the four ABR Wave-V level conditions. The results for the 100-dB-peSPL ABR condition are visualised as regression plots in Fig. 10. Only data points above the statistical noise floor were included and data points below the statistical noise floor were
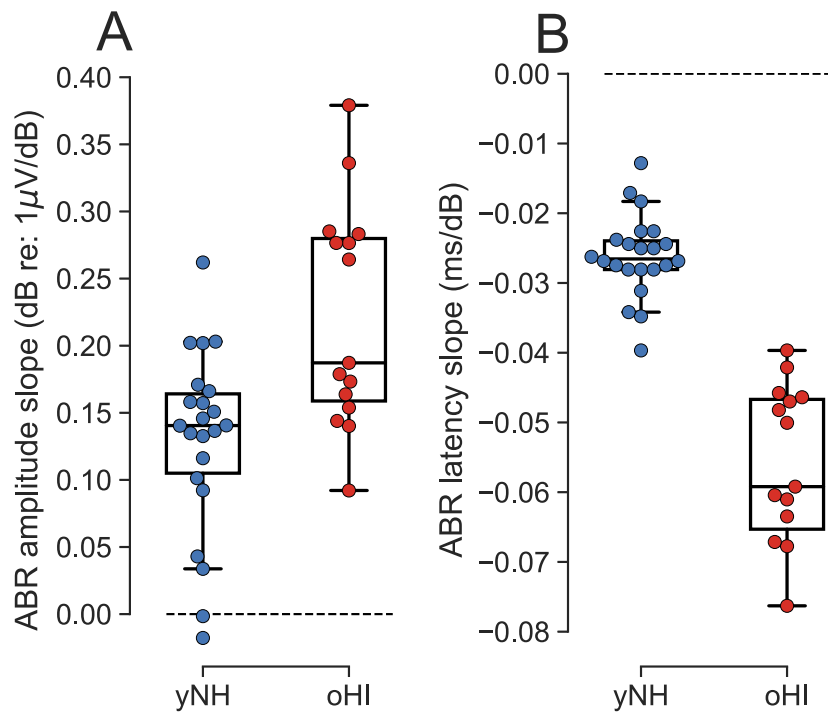

Fig. 8. Boxplots and individual data points of the ABR Wave-V (A) amplitude and (B) latency slopes (slope of a straight line fitted through all four increasing peSPL conditions) for both groups ( $\mathrm{yNH}, \mathrm{oHI})$. More positive amplitude slopes represent faster increasing amplitudes with increasing peSPL. More negative latency slopes represent faster decreasing latencies with increasing peSPL 


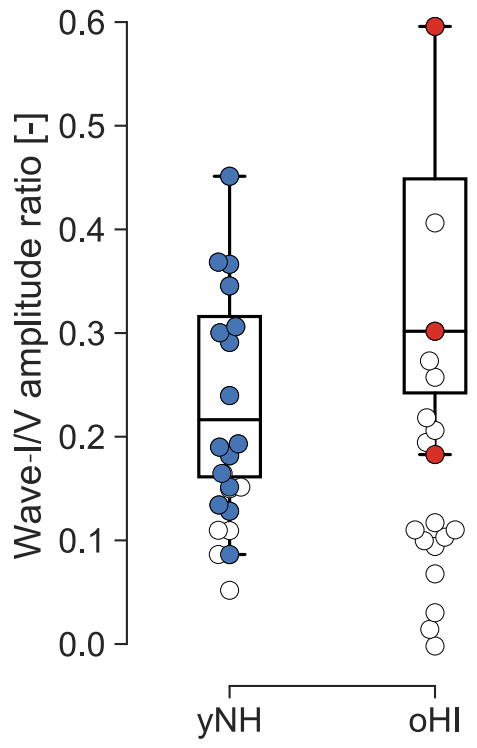

Fig. 9. Boxplots and individual data points of the ABR Wave-I/V amplitude ratio for both participant groups (yNH, oHI). White markers indicate ratios based on one or both wave peaks below the statistical noise floor.

marked with squares. As expected, a significant correlation $(\mathrm{r}=0.45 ; \mathrm{p}=0.0466, \mathrm{~N}=22)$ was found for the $\mathrm{yNH}$ participants in the $\mathrm{BB}$ group $\left(\mathrm{f}_{\mathrm{m}}: 120 \mathrm{~Hz}\right.$ ), which elicited the strongest EFRs due to the broader excitation pattern on the basilar membrane. A similar but weaker trend was also observed for the PT-EFR data. Even the oHI participants showed a positive trend in the BB condition which was mostly driven by two participants with large EFRs. Without those two data points, the correlation disappeared completely $(\mathrm{r}=0.03 ; \mathrm{p}=0.9358, \mathrm{~N}=14)$. No regression was fitted for the oHI pure-tone condition due to a lack of data points above the statistical noise floor. The BB-480-Hz EFRs for the yNH participants with responses above the statistical noise floor (14 out of 20), correlated significantly with three ABR level conditions ( $80 \mathrm{~dB}: \rho=0.59$, $\mathrm{p}=0.0260 ; \quad 90 \mathrm{~dB}: \quad \mathrm{r}=0.66, \quad \mathrm{p}=0.0097 ; \quad 100 \mathrm{~dB}: \mathrm{r}=0.56$, $\mathrm{p}=0.0306 ; \mathrm{N}=14$ ). The relation between the $\mathrm{BB}-480-\mathrm{Hz}$ EFR and the $100 \mathrm{~dB}$-peSPL ABR is depicted in the first panel of Fig. 10 (black markers).

For the relative metrics (ABR amplitude slope and EFR slope), we expected a positive linear relationship for the yNH participants, reflecting a positive covariation of neural recruitment with temporal coding fidelity ability. However, the data did not show any significant or consistent trends (Fig. 11). The 0-dB MD EFR magnitudes showed a strong significant relation to the ABR amplitude slope (Fig. 12) but only for the yNH pure-tone condition $(r=0.05$; $\mathrm{p}=0.0008 ; \mathrm{N}=11)$.

\subsection{Age and metrics of peripheral hearing ability}

No significant correlation between age and any of the EEG metrics was observed within the $\mathrm{yNH}$ and oHI participant subgroups. Only the audiometric thresholds at $4 \mathrm{kHz}$ showed a weak correlation with age $(\rho=0.42 ; \mathrm{p}=0.0451)$ for the oHI group. When pooling both groups, and thereby increasing the age range from 14 to 77, all EFR measures in both main conditions (BB,PT) except for the PT $-8 \mathrm{~dB}$ modulation depth condition showed significant correlations with age $(0.0001<\mathrm{p} \leq 0.0008)$. This was not the case for

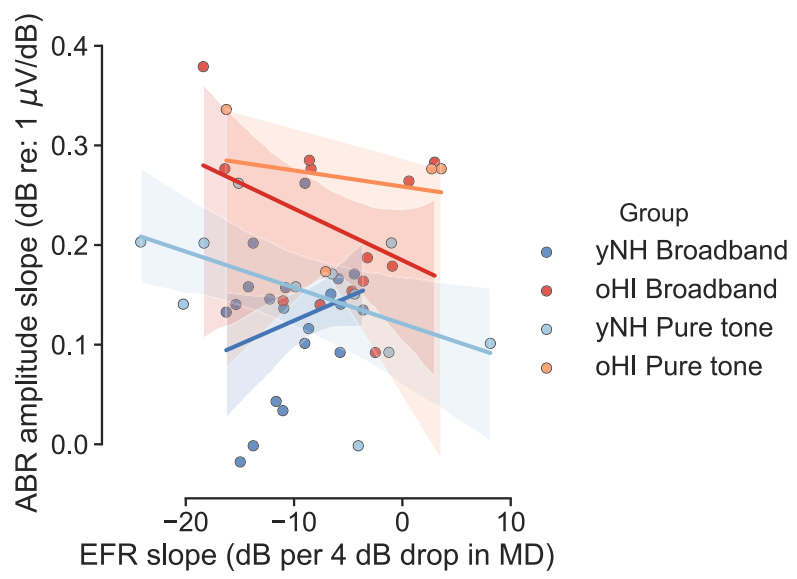

Fig. 11. Regression plots of the EFR SNR slopes (slope of a straight line fitted through all three modulation depth conditions) with the ABR Wave-V amplitude slopes for both main stimulus conditions (BB, PT) and participant groups (yNH, oHI). The shaded areas display the $95 \%$ confidence interval of the regression fit.

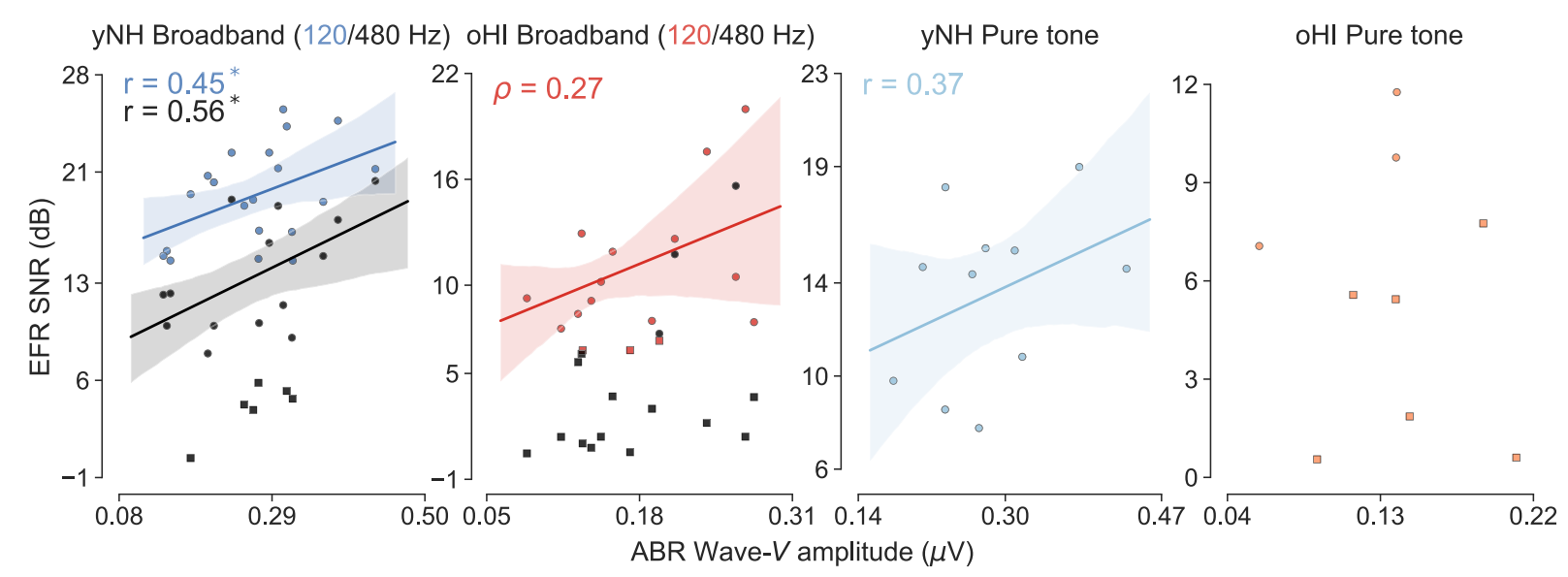

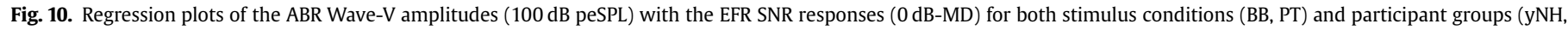

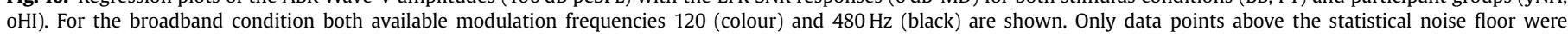

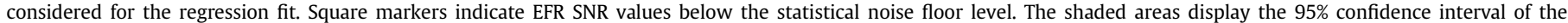

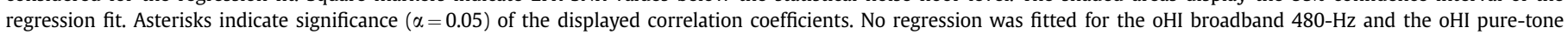
condition due to too few available data points. (For interpretation of the references to colour in this figure legend, the reader is referred to the Web version of this article.) 


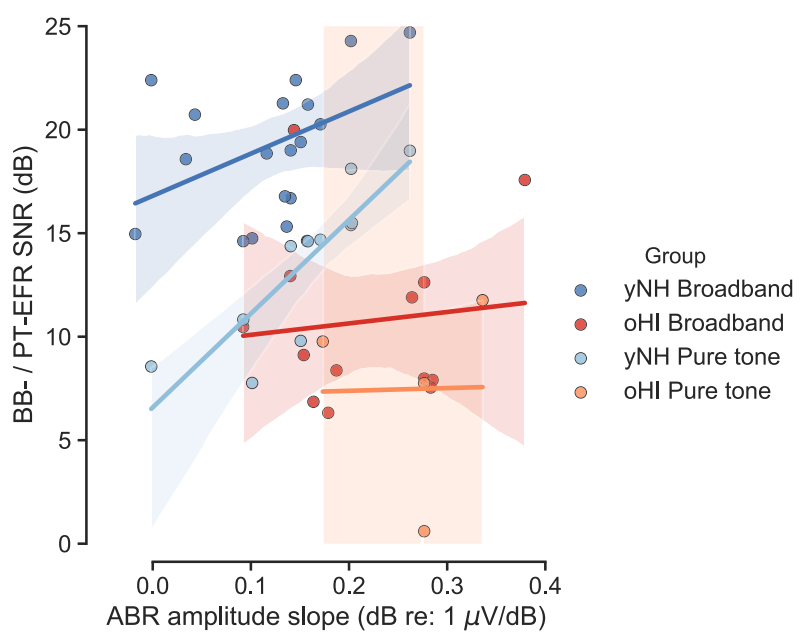

Fig. 12. Regression plots of the ABR Wave-V amplitude slope with the $0 \mathrm{~dB}-\mathrm{MD}$ EFR SNR magnitudes for both main stimulus conditions (BB, PT) and participant groups (yNH, oHI). The shaded areas display the 95\% confidence interval of the regression fit.

the relative EFR slopes, which did not reach significance. Correlations were not computed for the ABR Wave-I due to the small number of significant oHI data points. All tested ABR Wave-V level conditions $(0.0002 \leq \mathrm{p} \leq 0.0379)$ and the relative amplitude and latency slopes $(\mathrm{p}<0.0001)$ correlated with age across groups. This was also the case for the $4-\mathrm{kHz}$ audiogram $(\rho=0.82, \mathrm{p}<0.0001)$ and the DPOAE thresholds ( $\rho=0.58 ; \mathrm{p}=0.0001 ; \mathrm{N}=40$ ).

\section{Discussion}

We measured click-ABRs at different peSPLs and EFRs to a multitude of varying stimulus parameters to contrast response behaviour of absolute and relative electrophysiological metrics in young $\mathrm{NH}$ and older $\mathrm{HI}$ participants. In general, our results show that sensorineural hearing loss has an impact on subcortical EEG measures and that their interpretation strongly depends on the underlying types of impairments at play. Regarding our stated hypotheses in section 1.1 which were based on synaptopathy findings in $\mathrm{NH}$ participants, we observed the following:

1) oHI participants had reduced EFR magnitudes which, apart from one exception, did not relate to the indiviudal hearing thresholds. The results hence follow the hypothesis that the EFR magnitude is not systematically influenced by OHC loss and rather reflects the reduced temporal envelope coding ability in the oHI group.

2) The EFR slope metric did not show significant differences between groups and is likely not a robust marker for synaptopathy in listeners with impaired audiograms.

3) The oHI group had lower ABR Wave-V amplitudes in all four level conditions and the ABR Wave I was too weak to be reliably extracted in most oHI participants. Generally, the ABR amplitudes followed the synaptopathy hypothesis, but because oHI ABR latencies were at the same time increased, it is likely that both $\mathrm{OHC}$ and synaptopathy had a role in reducing the ABR amplitudes in this group.

4) oHI participants had steeper ABR amplitude and latency slopes than the yNH group. This observation contrasts the view that synaptopathy is the only factor driving these metrics.

5) The relationships between different measures of synaptopathy, yielded mixed results. Significant correlations were only observed between the 100-dB-peSPL yNH-ABR amplitude and the yNH BB-EFR for both modulation frequencies $(120 / 480 \mathrm{~Hz})$. Also the yNH 0-dB MD PT-EFR correlated to the ABR amplitude slope. Lastly, the EFR slope metric related to the EFR magnitudes but the modulation depth conditions that showed the strongest correlations differed between participant groups. These results suggest that EFR and ABR metrics of synaptopathy only relate in some of the conditions and in yNH listeners, whereas those relationships were absent in the oHI listeners where sensorineural hearing loss (mix of OHC deficits and synaptopathy) affects ABRs and EFRs differently.

6) All except for the relative EFR metrics, showed strong correlations with age when pooling data from both participant groups. However, no significant age correlations were found within the groups, suggesting that within a group the measures were not sensitive enough to isolate age-related synaptopathy from the other factors at play, while general age effects were observed on an overall group level.

These main findings are further discussed in the following sections.

\subsection{Effects of hearing threshold elevation}

\subsubsection{Consequences for the EFR}

The coding of temporal fluctuations at moderate-to-high levels is believed to be facilitated by the interplay of nerve fibres with high dynamic ranges (low-SR) and unsaturated off-frequency fibres (Encina-Llamas et al., 2019). Hearing, as tested using the classical audiogram, only requires a small number of highly sensitive fibres (high-SR) to detect the presence of a sound (Liberman and Kujawa, 2017). A relation between audiometric thresholds and the EFR, the latter reflecting temporal coding fidelity in the early stages of the auditory system, was not expected nor found within our two participant groups (with the oHI PT-EFR condition as an exception). Nevertheless, when treating the participant sample as a whole, our data show that audiometric hearing loss does have a significant effect on EFR strength. Observing the effect only when pooling the subjects, but not within the yNH or oHI group could be explained on the basis of co-occurring synaptopathy and $\mathrm{OHC}$ deficits in oHI listeners. Hence, within a group of listeners with similar audiometric hearing loss, individual differences in EFR magnitudes could reflect individual degrees of synaptopathy. At least for the PT-EFR condition, simulations with a model of the human auditory periphery predict that individual differences in NH and HI EFRs are most impacted by different degrees of synaptopathy and to a lesser extent by OHC deficits (Verhulst et al., 2018a,b). This does of course not exclude the possibility that other differences between the groups (e.g. age-related changes) might also have contributed to the observed effects. EFRs were shown to reduce with decreasing modulation depth in both groups (Fig. 2), implying a similar variation of the ability to encode weak temporal cues in the yNH and oHI auditory system. Our findings corroborate observations in humans (Dimitrijevic et al., 2016) and in animal models, which also show reduced EFRs with age/noise exposure (Parthasarathy et al., 2014; Shaheen et al., 2015; Parthasarathy and Kujawa, 2018).

\subsubsection{Relationship between SPL and the modulated neural firing rate}

We contrasted subcortical EEG metrics of yNH and oHI participants recorded at the same SPLs to mimic every-day listening conditions and to capture effects of $\mathrm{OHC}$ and neural deficits. A sensation level (SL) compensation would have minimised the effect of OHC loss. The use of the SPL approach additionally avoids the non-trivial challenge of applying an appropriate individual hearing sensitivity compensation method. Perceptual approaches based on 
the ABR/EFR stimulus threshold or on the audiogram, can introduce additional variability in the measures if the cancellation is imperfect.

It is known that the modulated firing rate of AN fibres in response to a SAM tone is not monotonously increasing with SPL, but instead shows a bell shape with a level-dependent maximum, the position of which depends on the fibre type (low-SR or high-SR; Joris and Yin, 1992; Kale and Heinz, 2010; Verhulst et al., 2018a,b). Given a sufficient number of intact nerve fibres, this means that a reduction of SPL within a certain range could actually lead to an increase in neural synchronisation as represented by the EFR. If the amplification capacity of the OHCs is impaired, thereby reducing the effective drive to the IHC/AN complex, this effect of increased modulated firing rates for reduced SPL input, might also be observable in EFRs to medium-to-high stimulus SPLs. This mechanism might partly explain the significantly increasing yNH-EFR magnitudes in response to the 5-dB stimulus level decrease for otherwise identical narrowband conditions (Fig. 5A). The same mechanism could also have been the basis of the significant positive correlation between oHI PT-EFRs and the audiometric thresholds at $4 \mathrm{kHz}$. In line with our findings, enhanced sensitivity to amplitude modulation, after the introduction of noise-induced hearing loss, was also reported for chinchillas for carrier frequencies above $2 \mathrm{kHz}$ and similar modulation frequencies (Zhong et al., 2014). The authors of the latter study interpreted this as a compensatory mechanism of the periphery and/or central structures.

\subsubsection{Consequences for the $A B R$}

While showing good retest-reliability at medium SPL in $\mathrm{NH}$ participants (Prendergast et al., 2018), the Wave I in humans is harder to measure than the Wave $\mathrm{V}$ and varies greatly in amplitude and latency between participants using the vertex configuration (Beattie, 1988; Lauter and Loomis, 1988; Trune et al., 1988; Mehraei et al., 2016). Especially in the oHI group, the recorded ABR waveforms were mostly too weak to reliably extract Wave-I information. As computational modelling work has predicted that the ABRWave-I amplitudes are reduced by synaptopathy and $\mathrm{OHC}$ loss (Verhulst et al., 2016) we suspect that this may be the reason for the low oHI Wave-I amplitudes. On the other hand, the ABR Wave V is very robust in humans and is believed to be generated in the lateral lemniscus and inferior colliculus (Møller and Jannetta, 1985; Melcher et al., 1996). The ABR Wave V was clearly detectable from our recordings even for oHI participants (Fig. 6/7). In agreement with earlier observations (Konrad-Martin et al., 2012), elevated hearing thresholds resulted in reduced ABR Wave-V amplitudes in all tested SPL conditions in comparison to the yNH-ABRs.

Even though the Wave $\mathrm{V}$ is a marker of early auditory processing, it cannot deliver identical information to the Wave I regarding AN processing. It is therefore important to consider whether the use of Wave V can be discussed in the context of synaptopathy. At least several aspects of Wave-I characteristics are reflected in Wave V. For example, changes in ABR Wave-I amplitude have been shown to be mirrored in the Wave- $\mathrm{V}$ latency shift for varying masking noise levels while ABR Wave-I and V amplitudes did not relate in the quiet condition without masking noise (Mehraei et al., 2016). It was also reported that the latencies of both waves covary for frequency contributions above $2 \mathrm{kHz}$ (Don and Eggermont, 1978). Based on this observation, and the particularly strong vulnerability of high-frequency cochlear regions to OHC loss, the increased Wave- $\mathrm{V}$ latencies in oHI participants might be interpreted as a neural marker for high-frequency OHC loss.

Even though a reduction in the number of nerve fibres as a consequence of cochlear synaptopathy cannot be excluded as a possible contributor, as functional ABR model simulations show that both, synaptopathy and OHC loss, reduce the Wave-V amplitude (Verhulst et al., 2016) in the absence of compensatory brainstem gain mechanisms (Chambers et al., 2016; Möhrle et al., 2016) our Wave-V results suggest that their reduced amplitudes were predominantly affected by $\mathrm{OHC}$ loss.

\subsection{Effects of stimulus features}

\subsubsection{Effects of bandwidth on the EFR}

Our results show clear differences as a consequence of manipulations to the stimulus bandwidth in both groups (Fig. 5A). An expected significant decrease in EFR magnitude with stimulus bandwidth reduction was only observed in the yNH group and might reflect a more restricted synchronised firing of the spiral ganglion cells and subsequent neural processing stages in the ascending auditory pathway. Unfortunately, the PT conditions did not result in maximising individual differences, but rather led to a greater number of EFRs below the statistical noise floor level for the oHI participants. The NB stimulus (at least at $75 \mathrm{~dB}$ SPL) was able to evoke significant responses in both groups while retaining good frequency specificity. However, without the use of off-frequency masking, the relative contributions of off-frequency fibres to the NB-EFR response remains unclear. The lack of sensitivity to the stimulus-bandwidth changes in the oHI-EFRs might be attributed to the wider auditory filters as a consequence of $\mathrm{OHC}$ loss. A wider band of frequencies falls within a certain filter, thereby reducing the frequency selectivity and associated frequency-specific coding. In addition to the consequences of OHC loss, a reduction of the number of nerve fibres might lead to a diminished ability of the brain to code subtle stimulus envelope changes robustly enough to be picked up by the scalp EEG.

\subsubsection{Effects of modulation frequency and the EFR generators}

Increasing the stimulus modulation frequency reduced the EFR significantly and similarly for both groups (Fig. 5B). Our results corroborate the EFR reductions observed for higher modulation frequencies in temporal modulation transfer functions (Purcell et al., 2004). Because we compensated for the frequency dependent noise floor $(\sim 1 / \mathrm{f})$ by using the EFR SNR metric, the effect of modulation frequency on the EFR is likely attributed to the frequency-dependent constructive and destructive phase interferences of multiple neural generators thought to be responsible for the EFR generation (Tichko and Skoe, 2017). Even though it is harder to measure the EFR to a $480-\mathrm{Hz}$ modulator reliably, they might reflect more peripheral generators than the IC and offer a better proxy measure for synaptopathy (Shaheen et al., 2015). Our finding that the BB-EFR at $480 \mathrm{~Hz}$ for yNH participants is more strongly related to the ABR Wave-V measure compared to the BBEFR at $120 \mathrm{~Hz}$ (Fig. 10) is in line with this idea. Unfortunately, higher modulation frequencies come at the cost of missing data points due to generally weaker responses in the oHI group. Lastly, we note that the argument about more peripheral sources for higher modulation frequencies (Purcell et al., 2004) only holds for noise carriers. The use of a $480-\mathrm{Hz}$ modulator on a pure tone carrier yields resolved AM side-bands (Kohlrausch et al., 2000), which result in an across-frequency spectral representation of the AM stimulus rather than a temporal envelope cue associated with AM side-band frequencies that fall within a single auditory filter.

\subsection{Applicability of relative metrics in the normal and impaired auditory system}

\subsubsection{The EFR slope}

The yNH BB-EFR slopes (Fig. 3) were comparable to those reported in a previous study (for a $100 \mathrm{~Hz}$ transposed $4-\mathrm{kHz}$ pure tone 
carrier; Bharadwaj et al., 2015) which related shallower EFR slopes to better AM and ITD detection thresholds as well as performance in a selective attention task. Different from that study, we did not use off-frequency notched noise masking. Consequently, the more widely spread slope values in the PT compared to the BB condition could in part be explained by an additional off-frequency contribution to the PT-EFRs, whose extent might vary between listeners. We did not observe significant differences in EFR slopes between the $\mathrm{yNH}$ and oHI group, in contrast to what we hypothesised. This challenges the interpretation of the EFR slope metric in light of synaptopathy, as it is expected that synaptopathy occurs before OHC loss sets in with age (Sergeyenko et al., 2013; Parthasarathy and Kujawa, 2018). However, the potential contribution of offfrequency fibres to the PT-EFRs might have affected the slope metrics in the two groups differently and thereby washed out the expected effects caused by synaptopathy; i.e. the increased cochlear filter width in the oHI population could have yielded stronger off-frequency contributions compared to the yNH group. We therefore conclude that factors such as OHC loss and synaptopathy have different impacts on the EFR slope metric and this metric is hence not exclusively sensitive to synaptopathy. The potential presence of neural and sensory factors of peripheral hearing loss on the EFR slope metric does not allow for a clear interpretation of this metric in the oHI group. The few (mainly oHI participants) showing positive slope values reflect the susceptibility of the EFR slope metric to outliers and it is not clear whether a straightline fit through the points best describes the AM depth function (Dimitrijevic et al., 2016).

\subsubsection{The ABR amplitude and latency slope}

Our data revealed shallower ABR amplitude and latency slopes for the yNH group in contrast to the oHI group for peSPL levels between 70 and $100 \mathrm{~dB}$ (Fig. 8). At those stimulus levels, high-SR fibres will already have reached their maximum discharge rate and any further increase in amplitude of the brainstem response might be due to the contributions of low-SR fibres that show higher saturation levels (Liberman, 1978; Heinz and Young, 2004). A steep slope in the yNH group might therefore reflect a healthy SR-fibre population consisting of both low and high SR fibres (Furman et al., 2013). However, in contrast to our expectations based on the synaptopathy-hypothesis, the oHI group actually showed steeper ABR Wave-V amplitude growth. OHC loss therefore seems to play the dominating role in determining the amplitude slope, by showing a recruitment of additional high-frequency channels with increasing peSPLs which result in stronger synchronised responses and steeper amplitude slopes. This broadening of peripheral auditory filters at high SPLs results in a more basally peaking excitation pattern (Ren, 2002). Increased Wave-V latencies and steeper ABR latency slopes with age, lifetime noise exposure, or as a function of audiometrically sloping hearing loss have also been observed in other studies (e.g. Burkard and Sims, 2002; Prendergast et al., 2017). The yNH latency slopes were less steep than those reported for the oHI group and corroborate slope values reported in many other studies (e.g. Don and Eggermont, 1978; Eggermont and Don, 1980; Dau, 2003; Mehraei et al., 2016). At 100 dB SPL, the latencies of both groups converged and no longer differed significantly (Fig. 7). At this high stimulus level, latency and amplitude actually showed a significant negative relationship in the oHI group indicating that at high peSPL, the ABR Wave-V amplitude is proportional to the extent with which auditory filters broaden by $\mathrm{OHC}$ loss. These results suggest that increased stimulus levels can restore the oHI-ABR latency to normal while the amplitudes remain smaller in this group (Neely et al., 2003; Lewis et al., 2015).

The impossibility of restoring the ABR amplitude at higher stimulus levels might be indicative of additional neural fibre loss or a less synchronised response in the oHI group. Participants with shorter latency values at high peSPLs (normal OHC function) whilst also showing low amplitude values (low synchronised neural response) might be impacted particularly strongly by cochlear synaptopathy (Verhulst et al., 2016). This idea stands in contrast to the hypothesis of a central gain compensation mechanism (Schaette and McAlpine, 2011; Chambers et al., 2016). Due to the low number of extractable Wave-I data for the oHI group, we were not able to do a more thorough investigation of such gain mechanisms based on the Wave-I/V ratio (see Fig. 9). Given the degraded auditory input for the oHI participants, a smaller Wave-I/V ratio would have been expected (Schaette and McAlpine, 2011) while the few available data points do not confirm this. This somewhat relates to another study which did not find differences in Wave-I/V ratios between groups with and without tinnitus (Guest et al., 2017). A missing gain effect in the oHI group might also partially be explained when considering age as a factor. It was recently shown that compensatory gain mechanisms were only present in young but not old rats in a similar SPL range as was considered here (Möhrle et al., 2016).

\subsection{Interrelation of EFR and $A B R$ metrics}

Contrasting the normal and impaired auditory system, we found a significant relation between the yNH-ABR Wave $\mathrm{V}$ and yNH-EFR amplitudes for the 120 and $480 \mathrm{~Hz}$ modulated $\mathrm{BB}$ condition (Fig. 10). As the neurons responsible for coding temporal stimulus modulations are presumably only a subset of all neurons responding to the ABR click stimulus, this relationship between overlapping neural generators was expected. Interestingly, this relationship breaks down in participants with peripheral hearing loss. Other authors who reported similar relationships between the EFR and ABR in rats interpret this as a decoupling of phasic and tonic synchrony caused by changing ratios of neurons representing the different stimuli with age (Parthasarathy et al., 2014). Another potential mechanism that could have caused the missing relation between the oHI-ABR and oHI-EFR is the loss of frequency selectivity that would allow more neurons to respond to the AM stimuli, without altering the neural contributions to the broadband click ABR.

The missing correlations between the EFR slope as a normalised measure of temporal coding fidelity and the ABR amplitude slope as a normalised metric of neural recruitment in both groups was unexpected, as other studies have suggested that both metrics independently relate to synaptopathy expression (e.g. Furman et al., 2013; Bharadwaj et al., 2014). The missing relationships could have several reasons. The ABR amplitude slopes in the $\mathrm{yNH}$ group showed very homogenous values and therefore very little variance. The degree to which the participants in this study are affected by nerve fibre loss might just be too small to be uncovered by the presented metrics. As described earlier, the EFR slope is hard to interpret in the presence of sensorineural hearing loss. Similarly, the oHI-ABR amplitude slope might primarily reflect OHC loss which is not directly linked to cochlear synaptopathy.

\subsection{Suitability for clinical applications/Recommendations}

Good SNRs and low variability are crucial for the adoption of a metric, especially in a diagnostic context. To restrain the huge parameter space for potential diagnostic metrics of synaptopathy, we will discuss important design considerations and recommendations based on our findings to guide future research.

In contrast to other groups (e.g. Liberman et al., 2016; Bramhall et al., 2017) who recorded Wave I with gold foil tiptrode electrodes in NH groups, our ABR Wave-I amplitudes were recorded with a 
conventional EEG setup and were often too weak to be picked up reliably (Fig. 6). Due to its difficult ascertainment and high variability using standard procedures, this measure is less attractive for diagnostic purposes in the context of synaptopathy and has already been shown to be an unreliable predictor for hearing-in-noise performance (Lobarinas et al., 2017). A big caveat of the use of EFRs in a clinical context is the low signal strength, primarily for the oHI group. Particularly, for the pure-tone conditions, lower modulation depths and high modulation frequencies yielded EFRs that were below the statistical noise floor and therefore show low reliability. To increase the detection sensitivity of the desired signals using EEG, we recommend using sufficiently high stimulus levels, especially for oHI participants. Presenting the stimuli at sensation level will help in that respect, but will not allow for the analysis of the OHC aspect of sensorineural hearing loss. Additionally, the signal carrier type was crucial to achieve good SNRs in the EFR recordings. The use of narrowband noise as opposed to a pure-tone carrier resulted in increased magnitudes while sustaining the frequency selectivity of the EFR (see Fig. 5). We therefore encourage the use of band-limited noise carriers and additionally suggest to adapt the stimulus envelope shape to further increase response magnitudes (see John et al., 2002, 2003). The choice of modulation frequency for EFRs depends on the specific research question (i.e., higher frequencies for more peripheral generator sources, Purcell et al., 2004) but due to the low-pass characteristics of the skull, the adoption of higher frequencies is generally penalized with lower response magnitudes. General guidance regarding the optimal modulation frequency can be found in Tichko and Skoe (2017). Long duration EFR stimuli might also be desired because this would allow for a separate analysis of onset/offset and steadystate portions of the signal, whereas we considered the entire 600ms waveform in our analysis. We would also like to encourage future research to pay close attention to the neural noise floor in the EFR recordings and to use suitable techniques like the subtraction of the noise floor or the EFR SNR metric used here, to take differences in noise floor levels between groups into account (also see Purcell et al., 2004; Picton et al., 2005).

Though theoretically superior, the relative metrics we considered in this study seem very susceptible to the influence of noisy data points, especially for linear fits and vary considerably between participants as they incorporate the variance of multiple data points. Additionally, as these metrics are based on data points with varying stimulus parameters (e.g. level, modulation depth) it is unknown if they are comparable in the excitation patterns they elicit on the basilar membrane or in their neural generators. Specifically for the ABRs, we would also suggest to use lower repetition rates than the $33.3 \mathrm{~Hz}$ we used, to increase the resulting amplitudes/SNR and to carefully consider the advantages and disadvantages of single vs. alternating polarities (e.g. Picton, 2010). Even though it is not easy to recruit older $\mathrm{NH}$ participants, we strongly encourage researchers to also include an old $\mathrm{NH}$ control group to isolate the effects of age and $\mathrm{OHC}$ loss on the measured responses and be able to better assess the effect cochlear synaptopathy has on electrophysiological metrics.

\subsection{Limitations}

As the interpretation of individual subcortical EEG metrics are multifaceted, ranging from sensory to neural contributors and other age related changes, the present study can only shed light on how these metrics, believed to relate to the neural processing in the early auditory pathway, behave in two extreme groups of young normal hearing and elderly hearing impaired participants. Possible explanations for the relationships between measures were discussed based on recent findings in the synaptopathy field but cannot unambiguously be supported by the present data. One of the overall conclusions of this study is, that the neural signal strength and reliability of data points are greatly diminished in the oHI group when testing $\mathrm{yNH}$ and oHI participants on the same set of EEG metrics. Additionally, the uncertainty in the interpretation of data that fell below the estimated statistical noise floor must be handled with care, even though the data points had positive SNRs. To capture a wide range of synaptopathy profiles, from very mild to advanced synaptic loss, we recruited participants from a broad age and hearing status range. This participant selection was instrumental in showing the wide range of outcomes that can be expected from a clinical population but reduces the interpretability of individual metrics.

\section{Summary and conclusion}

This study recorded multiple subcortical EEG measures in young $\mathrm{NH}$ and older $\mathrm{HI}$ participant groups to investigate how these metric behave in the presence of sensory and neural hearing deficits. We showed that EFRs and ABRs can be recorded in aged participants with sloping sensorineural hearing loss for certain stimulus conditions. Both ABRs and EFRs showed lower amplitudes in the oHI group and were only significantly related in the $\mathrm{yNH}$ group, thereby replicating the findings from numerous other human and animal studies. The use of relative ABR and EFR metrics can provide valuable insight into the underlying hearing deficit mechanisms but the high degree of variability (especially in the oHI group) allows interpretations only on a group level and is, in the current design, less suitable for individual diagnostics. This study reported what, realistically, can be expected when applying and combining different established electrophysiological metrics to the normal and aged impaired auditory system. Our findings can help guide future developments of electrophysiological measures of cochlear synaptopathy toward becoming a reliable and informative clinical tool.

\section{Declaration of conflicting interests}

The authors declared no potential conflicts of interest with respect to the research, authorship, and/or publication of this article.

\section{Funding}

The authors disclosed receipt of the following financial support for the research, authorship, and/or publication of this article: This work was supported by the DFG Cluster of Excellence EXC 1077/1 "Hearing4all".

\section{Acknowledgement}

The authors thank Anoop Jagadeesh, Frauke Ernst, Marvin Schmidt and Moritz Wächtler for help with the data collection, Dr. Manfred Mauermann for the help with DPOAE analyses and setup, and Dr. Vasilkov for feedback on the manuscript.

\section{Appendix A. Supplementary data}

Supplementary data to this article can be found online at https://doi.org/10.1016/j.heares.2019.07.001.

\section{References}

Beattie, R.C., 1988. Interaction of click polarity, stimulus level, and repetition rate on the auditory brainstem response. Scand. Audiol. 17 (2), 99-109. https://doi.org/ 
10.3109/01050398809070698.

Bharadwaj, H.M., Masud, S., Mehraei, G., Verhulst, S., Shinn-Cunningham, B.G., 2015 Individual differences reveal correlates of hidden hearing deficits. J. Neurosci. 35 (5), 2161-2172. https://doi.org/10.1523/JNEUROSCI.3915-14.2015.

Bharadwaj, H.M., Verhulst, S., Shaheen, L., Liberman, M.C., Shinn-Cunningham, B.G., 2014. Cochlear neuropathy and the coding of supra-threshold sound. Front Syst. Neurosci. 8, 26. https://doi.org/10.3389/fnsys.2014.00026.

Boege, P., Janssen, T., 2002. Pure-tone threshold estimation from extrapolated distortion product otoacoustic emission $\mathrm{I} / \mathrm{O}$-functions in normal and cochlear hearing loss ears. J. Acoust. Soc. Am. 111 (4), 1810-1818. https://doi.org/10.1121/ 1.1460923.

Bourien, J., Tang, Y., Batrel, C., Huet, A., Lenoir, M., Ladrech, S., Wang, J., 2014. Contribution of auditory nerve fibers to compound action potential of the auditory nerve. J. Neurophysiol. 112 (5), 1025-1039. https://doi.org/10.1152/ jn.00738.2013.

Bramhall, N., Beach, E.F., Epp, B., Le Prell, C.G., Lopez-Poveda, E.A., Plack, C.J., Canlon, B., 2019. The search for noise-induced cochlear synaptopathy in humans: mission impossible? Hear. Res. 377, 88-103. https://doi.org/10.1016/ j.heares.2019.02.016.

Bramhall, N.F., Konrad-Martin, D., McMillan, G.P., Griest, S.E., 2017. Auditory brainstem response altered in humans with noise exposure despite normal outer hair cell function. Ear Hear. 38 (1), e1-e12. https://doi.org/10.1097/ AUD.0000000000000370.

Burkard, R.F., Sims, D., 2002. A comparison of the effects of broadband masking noise on the auditory brainstem response in young and older adults. Am. J. Audiol. 11 (1), 13-22. https://doi.org/10.1044/1059-0889(2002/004.

Carney, L.H., 2018. Supra-threshold hearing and fluctuation profiles: implications for sensorineural and hidden hearing loss. J. Assoc. Res. Otolaryngol.: JARO 19 (4), 331-352. https://doi.org/10.1007/s10162-018-0669-5.

Chambers, A.R., Resnik, J., Yuan, Y., Whitton, J.P., Edge, A.S., Liberman, M.C., Polley, D.B., 2016. Central gain restores auditory processing following nearcomplete cochlear denervation. Neuron 89 (4), 867-879. https://doi.org/ 10.1016/j.neuron.2015.12.041.

Dau, T., 2003. The importance of cochlear processing for the formation of auditory brainstem and frequency following responses. J. Acoust. Soc. Am. 113 (2), 936-950. https://doi.org/10.1121/1.1534833.

Debener, S., Thorne, J., Schneider, T.R., Viola, F.C., 2010. Using ICA for the analysis of multi-channel EEG data. In: Ullsperger, M., Debener, S. (Eds.), Simultaneous EEG and fMRI: Recording, Analysis, and Application. Oxford University Press, pp. 121-133.

Dimitrijevic, A., Alsamri, J., John, M.S., Purcell, D., George, S., Zeng, F.-G., 2016. Human envelope following responses to amplitude modulation: effects of aging and modulation depth. Ear Hear. 37 (5), e322-e335. https://doi.org/10.1097/ AUD.0000000000000324.

Don, M., Eggermont, J.J., 1978. Analysis of the click-evoked brainstem potentials in man using high-pass noise masking. J. Acoust. Soc. Am. 63 (4), 1084-1092. https://doi.org/10.1121/1.381816.

Eggermont, J.J., Don, M., 1980. Analysis of the click-evoked brainstem potentials in humans using high-pass noise masking. II. Effect of click intensity. J. Acoust. Soc. Am. 68 (6), 1671-1675. https://doi.org/10.1121/1.385199.

Encina-Llamas, G., Harte, J.M., Dau, T., Shinn-Cunningham, B., Epp, B., 2019. Investigating the effect of cochlear synaptopathy on envelope following responses using a model of the auditory nerve. J. Assoc. Res. Otolaryngol.: JARO. https:// doi.org/10.1007/s10162-019-00721-7.

Fernandez, K.A., Jeffers, P.W.C., Lall, K., Liberman, M.C., Kujawa, S.G., 2015. Aging after noise exposure: acceleration of cochlear synaptopathy in 'recovered' ears. J. Neurosci. 35 (19), 7509-7520. https://doi.org/10.1523/JNEUROSCI.513814.2015.

Festen, J.M., Plomp, R., 1983. Relations between auditory functions in impaired hearing. J. Acoust. Soc. Am. 73 (2), 652-662. https://doi.org/10.1121/1.388957.

Fulbright, A.N.C., Le Prell, C.G., Griffiths, S.K., Lobarinas, E., 2017. Effects of recreational noise on threshold and suprathreshold measures of auditory function. Semin. Hear. 38 (4), 298-318. https://doi.org/10.1055/s-0037-1606325.

Furman, A.C., Kujawa, S.G., Liberman, M.C., 2013. Noise-induced cochlear neuropathy is selective for fibers with low spontaneous rates. J. Neurophysiol. 110 (3), 577-586. https://doi.org/10.1152/jn.00164.2013.

Glasberg, B.R., Moore, B.C., 1986. Auditory filter shapes in subjects with unilateral and bilateral cochlear impairments. J. Acoust. Soc. Am. 79 (4), 1020-1033. https://doi.org/10.1121/1.393374.

Gorga, M.P., Worthington, D.W., Reiland, J.K., Beauchaine, K.A., Goldgar, D.E., 1985 Some comparisons between auditory brain stem response thresholds, latencies, and the pure-tone audiogram. Ear Hear. 6 (2), 105-112. https://doi.org/10.1097/ 00003446-198503000-00008.

Gramfort, A., Luessi, M., Larson, E., Engemann, D.A., Strohmeier, D., Brodbeck, C., Hämäläinen, M., 2013. MEG and EEG data analysis with MNE-Python. Front Neurosci. 7, 267. https://doi.org/10.3389/fnins.2013.00267.

Gramfort, A., Luessi, M., Larson, E., Engemann, D.A., Strohmeier, D., Brodbeck, C., Hämäläinen, M.S., 2014. MNE software for processing MEG and EEG data. Neuroimage 86, 446-460. https://doi.org/10.1016/j.neuroimage.2013.10.027.

Guest, H., Munro, K.J., Prendergast, G., Howe, S., Plack, C.J., 2017. Tinnitus with a normal audiogram: relation to noise exposure but no evidence for cochlear synaptopathy. Hear. Res. 344, 265-274. https://doi.org/10.1016/ j.heares.2016.12.002

Heinz, M.G., Young, E.D., 2004. Response growth with sound level in auditory-nerve fibers after noise-induced hearing loss. J. Neurophysiol. 91 (2), 784-795. https://doi.org/10.1152/jn.00776.2003.

Hickox, A.E., Larsen, E., Heinz, M.G., Shinobu, L., Whitton, J.P., 2017. Translational issues in cochlear synaptopathy. Hear. Res. 349, 164-171. https://doi.org/ 10.1016/j.heares.2016.12.010.

Hind, S.E., Haines-Bazrafshan, R., Benton, C.L., Brassington, W., Towle, B. Moore, D.R., 2011. Prevalence of clinical referrals having hearing thresholds within normal limits. Int. J. Audiol. 50 (10), 708-716. https://doi.org/10.3109/ 14992027.2011.582049.

Humes, L.E., 2013. Understanding the speech-understanding problems of older adults. Am. J. Audiol. 22 (2), 303-305. https://doi.org/10.1044/1059-0889(2013 12-0066.

Humes, L.E., Kewley-Port, D., Fogerty, D., Kinney, D., 2010. Measures of hearing threshold and temporal processing across the adult lifespan. Hear. Res. 264 (1-2), 30-40. https://doi.org/10.1016/j.heares.2009.09.010.

ISO, 1990. Acoustics - Determination of Occupational Noise Exposure and Estimation of Noise-Induced Hearing Impairment. International Organization of Standardization, Switzerland. ISO 1999:1990.

John, M.S., Dimitrijevic, A., Picton, T.W., 2002. Auditory steady-state responses to exponential modulation envelopes. Ear Hear. 23 (2), 106-117. https://doi.org 10.1097/00003446-200204000-00004.

John, M.S., Dimitrijevic, A., Picton, T.W., 2003. Efficient stimuli for evoking auditory steady-state responses. Ear Hear. 24 (5), 406-423. https://doi.org/10.1097| 1.AUD.0000090442.37624.BE.

Johnson, E.W., 1970. Tuning forks to audiometers and back again. The Laryngoscope 80 (1), 49-68. https://doi.org/10.1288/00005537-197001000-00005.

Joris, P.X., Yin, T.C., 1992. Responses to amplitude-modulated tones in the auditory nerve of the cat. J. Acoust. Soc. Am. 91 (1), 215-232. https://doi.org/10.1121/ 1.402757.

Kale, S., Heinz, M.G., 2010. Envelope coding in auditory nerve fibers following noiseinduced hearing loss. J. Assoc. Res. Otolaryngol.: JARO 11 (4), 657-673. https:// doi.org/10.1007/s10162-010-0223-6.

Khouri, L., Lesica, N.A., Grothe, B., 2011. Impaired auditory temporal selectivity in the inferior colliculus of aged Mongolian gerbils. J. Neurosci. 31 (27) 9958-9970. https://doi.org/10.1523/JNEUROSCI.4509-10.2011.

Kobel, M., Le Prell, C.G., Liu, J., Hawks, J.W., Bao, J., 2017. Noise-induced cochlear synaptopathy: past findings and future studies. Hear. Res. 349, 148-154 https://doi.org/10.1016/j.heares.2016.12.008.

Kohlrausch, A., Fassel, R., Dau, T., 2000. The influence of carrier level and frequency on modulation and beat-detection thresholds for sinusoidal carriers. J. Acoust. Soc. Am. 108 (2), 723-734. https://doi.org/10.1121/1.429605.

Konrad-Martin, D., Dille, M.F., McMillan, G., Griest, S., McDermott, D., Fausti, S.A. Austin, D.F., 2012. Age-related changes in the auditory brainstem response J. Am. Acad. Audiol. 23 (1), 18-75. https://doi.org/10.3766/jaaa.23.1.3.

Kujawa, S.G., Liberman, M.C., 2009. Adding insult to injury: cochlear nerve degeneration after 'temporary' noise-induced hearing loss. J. Neurosci. 29 (45), 14077-14085. https://doi.org/10.1523/JNEUROSCI.2845-09.2009.

Kumar, G., Amen, F., Roy, D., 2007. Normal hearing tests: is a further appointment really necessary? J. R. Soc. Med. 100 (2), 66. https://doi.org/10.1177/ 014107680710000212

Kummer, P., Janssen, T., Arnold, W., 1998. The level and growth behavior of the $2 \mathrm{f1}-$ f2 distortion product otoacoustic emission and its relationship to auditory sensitivity in normal hearing and cochlear hearing loss. J. Acoust. Soc. Am. 103 (6), 3431-3444. https://doi.org/10.1121/1.423054.

Lauter, J.L., Loomis, R.L., 1988. Individual differences in auditory electric responses: comparisons of between-subject and within-subject variability. II. Amplitude of brainstem Vertex-positive peaks. Scand. Audiol. 17 (2), 87-92. https://doi.org/ 10.3109/01050398809070696.

Lewis, J.D., Kopun, J., Neely, S.T., Schmid, K.K., Gorga, M.P., 2015. Tone-burst auditory brainstem response wave $\mathrm{V}$ latencies in normal-hearing and hearing-impaired ears. J. Acoust. Soc. Am. 138 (5), 3210-3219. https://doi.org/10.1121/1.4935516.

Liberman, M.C., 1978. Auditory-nerve response from cats raised in a low-noise chamber. J. Acoust. Soc. Am. 63 (2), 442-455. https://doi.org/10.1121/1.381736.

Liberman, M.C., Epstein, M.J., Cleveland, S.S., Wang, H., Maison, S.F., 2016. Toward a differential diagnosis of hidden hearing loss in humans. PLoS One 11 (9) https://doi.org/10.1371/journal.pone.0162726 e0162726.

Liberman, M.C., Kujawa, S.G., 2017. Cochlear synaptopathy in acquired sensorineural hearing loss: manifestations and mechanisms. Hear. Res. 349, 138-147. https:// doi.org/10.1016/j.heares.2017.01.003.

Lin, H.W., Furman, A.C., Kujawa, S.G., Liberman, M.C., 2011. Primary neural degeneration in the Guinea pig cochlea after reversible noise-induced threshold shift J. Assoc. Res. Otolaryngol.: JARO 12 (5), 605-616. https://doi.org/10.1007/ s10162-011-0277-0.

Lobarinas, E., Spankovich, C., Le Prell, C.G., 2017. Evidence of 'hidden hearing loss' following noise exposures that produce robust TTS and ABR wave-I amplitude reductions. Hear. Res. 349, 155-163. https://doi.org/10.1016 j.heares.2016.12.009.

Makary, C.A., Shin, J., Kujawa, S.G., Liberman, M.C., Merchant, S.N., 2011. Age-related primary cochlear neuronal degeneration in human temporal bones. J. Assoc Res. Otolaryngol.: JARO 12 (6), 711-717. https://doi.org/10.1007/s10162-0110283-2.

Mauermann, M., 2013. Improving the usability of the distortion product otoacoustic emisssions (DPOAE)-sweep method: an alternative artifact rejection and noisefloor estimation. Proc. Meet. Acoust. 19 (1), 050054. https://doi.org/10.1121 1.4800902 .

Mehraei, G., Hickox, A.E., Bharadwaj, H.M., Goldberg, H., Verhulst, S., Liberman, M.C. 
Shinn-Cunningham, B.G., 2016. Auditory brainstem response latency in noise as a marker of cochlear synaptopathy. J. Neurosci. 36 (13), 3755-3764. https:// doi.org/10.1523/JNEUROSCI.4460-15.2016.

Melcher, J.R., Knudson, I.M., Fullerton, B.C., Guinan, J.J., Norris, B.E., Kiang, N.Y., 1996 Generators of the brainstem auditory evoked potential in cat. I. An experimental approach to their identification. Hear. Res. 93 (1-2), 1-27. https:/ doi.org/10.1016/0378-5955(95)00178-6.

Millman, K.J., Aivazis, M., 2011. Python for scientists and engineers. Comput. Sci. Eng. 13 (2), 9-12. https://doi.org/10.1109/MCSE.2011.36.

Mitchell, C., Phillips, D.S., Trune, D.R., 1989. Variables affecting the auditory brainstem response: audiogram, age, gender and head size. Hear. Res. 40 (1-2), 75-85. https://doi.org/10.1016/0378-5955(89)90101-9.

Möhrle, D., Ni, K., Varakina, K., Bing, D., Lee, S.C., Zimmermann, U., Rüttiger, L., 2016 Loss of auditory sensitivity from inner hair cell synaptopathy can be centrally compensated in the young but not old brain. Neurobiol. Aging 44, 173-184. https://doi.org/10.1016/j.neurobiolaging.2016.05.001.

Møller, A., Jannetta, P.J., 1985. Neural generators of the auditory brainstem response. In: Jacobson, J.T. (Ed.), The Auditory Brainstem Response. College Hill, San Diego, pp. 13-31.

Neely, S.T., Gorga, M.P., Dorn, P.A., 2003. Cochlear compression estimates from measurements of distortion-product otoacoustic emissions. J. Acoust. Soc. Am. 114 (3), 1499-1507. https://doi.org/10.1121/1.1604122.

Oliphant, T.E., 2007. Python for scientific computing. Comput. Sci. Eng. 9 (3), 10-20. https://doi.org/10.1109/MCSE.2007.58.

Parthasarathy, A., Bartlett, E.L., Kujawa, S.G., 2018. Age-related changes in neura coding of envelope cues: peripheral declines and central compensation. Neuroscience 407, 21-31. https://doi.org/10.1016/j.neuroscience.2018.12.007.

Parthasarathy, A., Datta, J., Torres, J.A.L., Hopkins, C., Bartlett, E.L., 2014. Age-related changes in the relationship between auditory brainstem responses and envelope-following responses. J. Assoc. Res. Otolaryngol: JARO 15 (4), 649-661. https://doi.org/10.1007/s10162-014-0460-1.

Parthasarathy, A., Kujawa, S.G., 2018. Synaptopathy in the aging cochlea: characterizing early-neural deficits in auditory temporal envelope processing. J. Neurosci. 38 (32), 7108-7119. https://doi.org/10.1523/JNEUROSCI.324017.2018.

Picton, T.W., 2010. Auditory brainstem responses: peaks along the way. In: Human Auditory Evoked Potentials. Plural Publishing, pp. 213-246.

Picton, T.W., Dimitrijevic, A., Perez-Abalo, M.-C., Van Roon, P., 2005. Estimating audiometric thresholds using auditory steady-state responses. J. Am. Acad. Audiol. 16 (3), 140-156. https://doi.org/10.3766/jaaa.16.3.3.

Pinheiro, J., Bates, D., DebRoy, S., Sarkar, D., R Core Team, 2017. Nlme: Linear and Nonlinear Mixed Effects Models (Version R Package. Retrieved from, version 3.1-131. https://CRAN.R-project.org/package $=$ nlme.

Plack, C.J., Léger, A., Prendergast, G., Kluk, K., Guest, H., Munro, K.J., 2016. Toward a diagnostic test for hidden hearing loss. Trends in Hearing 20. https://doi.org $10.1177 / 2331216516657466$

Prendergast, G., Guest, H., Munro, K.J., Kluk, K., Léger, A., Hall, D.A., Plack, C.J., 2017. Effects of noise exposure on young adults with normal audiograms I: Electrophysiology. Hear. Res. 344, 68-81. https://doi.org/10.1016/j.heares.2016.10.028.

Prendergast, G., Tu, W., Guest, H., Millman, R.E., Kluk, K., Couth, S., Plack, C.J., 2018 Supra-threshold auditory brainstem response amplitudes in humans: testretest reliability, electrode montage and noise exposure. Hear. Res. 364, 38-47. https://doi.org/10.1016/j.heares.2018.04.002.

Purcell, D.W., John, S.M., Schneider, B.A., Picton, T.W., 2004. Human tempora auditory acuity as assessed by envelope following responses. J. Acoust. Soc. Am. 116 (6), 3581-3593. https://doi.org/10.1121/1.1798354.

R Core Team, 2017. R: A Language and Environment for Statistical Computing. Retrieved from. https://www.R-project.org/.

Ren, T., 2002. Longitudinal pattern of basilar membrane vibration in the sensitive cochlea. Proc. Nat. Acad. Sci. U. S. A. 99 (26), 17101-17106. https://doi.org $10.1073 /$ pnas.262663699.

Richardson, B.D., Ling, L.L., Uteshev, V.V., Caspary, D.M., 2013. Reduced GABA(A) receptor-mediated tonic inhibition in aged rat auditory thalamus. J. Neurosci. 33 (3), 1218-1227. https://doi.org/10.1523/JNEUROSCI.3277-12.2013.

Russell, V., 2016. Least-squares means: the R package lsmeans. J. Stat. Softw. 69 (1),
1-33. https://doi.org/10.18637/jss.v069.i01

Schaette, R., McAlpine, D., 2011. Tinnitus with a normal audiogram: physiological evidence for hidden hearing loss and computational model. J. Neurosci. 31 (38), 13452-13457. https://doi.org/10.1523/JNEUROSCI.2156-11.2011.

Schimmel, H., 1967. The $(+)$ reference: accuracy of estimated mean components in average response studies. Science 157 (3784), 92-94. https://doi.org/10.1126/ science.157.3784.92.

Schmiedt, R.A., Mills, J.H., Boettcher, F.A., 1996. Age-related loss of activity of auditory-nerve fibers. J. Neurophysiol. 76 (4), 2799-2803. https://doi.org/ 10.1152/jn.1996.76.4.2799.

Sergeyenko, Y., Lall, K., Liberman, M.C., Kujawa, S.G., 2013. Age-related cochlear synaptopathy: an early-onset contributor to auditory functional decline. J. Neurosci. 33 (34), 13686-13694. https://doi.org/10.1523/JNEUROSCI.178313.2013.

Shaheen, L.A., Valero, M.D., Liberman, M.C., 2015. Towards a diagnosis of cochlear neuropathy with envelope following responses. J. Assoc. Res. Otolaryngol.: JARO 16 (6), 727-745. https://doi.org/10.1007/s10162-015-0539-3.

Stamataki, S., Francis, H.W., Lehar, M., May, B.J., Ryugo, D.K., 2006. Synaptic alterations at inner hair cells precede spiral ganglion cell loss in aging C57BL/6 mice. Hear. Res. 221 (1-2), 104-118. https://doi.org/10.1016/ j.heares.2006.07.014.

Tichko, P., Skoe, E., 2017. Frequency-dependent fine structure in the frequencyfollowing response: the byproduct of multiple generators. Hear. Res. 348, 1-15. https://doi.org/10.1016/j.heares.2017.01.014.

Trune, D.R., Mitchell, C., Phillips, D.S., 1988. The relative importance of head size, gender and age on the auditory brainstem response. Hear. Res. 32 (2-3), 165-174. https://doi.org/10.1016/0378-5955(88)90088-3.

Valderrama, J.T., Beach, E.F., Yeend, I., Sharma, M., Van Dun, B., Dillon, H., 2018. Effects of lifetime noise exposure on the middle-age human auditory brainstem response, tinnitus and speech-in-noise intelligibility. Hear. Res. 365, 36-48. https://doi.org/10.1016/j.heares.2018.06.003.

Valero, M.D., Burton, J.A., Hauser, S.N., Hackett, T.A., Ramachandran, R., Liberman, M.C., 2017. Noise-induced cochlear synaptopathy in rhesus monkeys (Macaca mulatta). Hear. Res. 353, 213-223. https://doi.org/10.1016/ j.heares.2017.07.003.

Verhulst, S., Altoè, A., Vasilkov, V., 2018a. Computational modeling of the human auditory periphery: auditory-nerve responses, evoked potentials and hearing loss. Hear. Res. 360, 55-75. https://doi.org/10.1016/j.heares.2017.12.018.

Verhulst, S., Ernst, F., Garrett, M., Vasilkov, V., 2018b. Supra-threshold psychoacoustics and envelope-following response relations: normal-hearing, synaptopathy and cochlear gain loss. Acta Acustica united Acustica 104 (5), 800-803. https://doi.org/10.3813/AAA.919227.

Verhulst, S., Jagadeesh, A., Mauermann, M., Ernst, F., 2016. Individual differences in auditory brainstem response wave characteristics: relations to different aspects of peripheral hearing loss. Trends in Hearing 20,1-20. https://doi.org/10.1177/ 2331216516672186.

Voytek, B., Kramer, M.A., Case, J., Lepage, K.Q., Tempesta, Z.R., Knight, R.T., Gazzaley, A., 2015. Age-related changes in 1/f neural electrophysiological noise. J. Neurosci. 35 (38), 13257-13265. https://doi.org/10.1523/JNEUROSCI.233214.2015

Wu, P.Z., Liberman, L.D., Bennett, K., de Gruttola, V., O'Malley, J.T., Liberman, M.C. 2018. Primary neural degeneration in the human cochlea: evidence for hidden hearing loss in the aging ear. Neuroscience 407, 8-20. https://doi.org/10.1016/ j.neuroscience.2018.07.053.

Yeend, I., Beach, E.F., Sharma, M., Dillon, H., 2017. The effects of noise exposure and musical training on suprathreshold auditory processing and speech perception in noise. Hear. Res. 353, 224-236. https://doi.org/10.1016/j.heares.2017.07.006.

Zhong, Z., Henry, K.S., Heinz, M.G., 2014. Sensorineural hearing loss amplifies neura coding of envelope information in the central auditory system of chinchillas. Hear. Res. 309, 55-62. https://doi.org/10.1016/j.heares.2013.11.006.

Zhu, L., Bharadwaj, H., Xia, J., Shinn-Cunningham, B., 2013. A comparison of spectral magnitude and phase-locking value analyses of the frequency-following response to complex tones. J. Acoust. Soc. Am. 134 (1), 384-395. https:// doi.org/10.1121/1.4807498. 